\title{
Vibrational-Exciton Couplings for the Amide I, II, III, and A Modes of Peptides
}

\author{
Tomoyuki Hayashi and Shaul Mukamel* \\ Department of Chemistry, University of California, Irvine, California 92697-2025, U.S.A.
}

Received: January 16, 2007; In Final Form: June 1, 2007

\begin{abstract}
The couplings between all amide fundamentals and their overtones and combination vibrational states are calculated. Combined with the level energies reported previously (Hayashi, T.; Zhuang, W.; Mukamel, S. J. Phys. Chem. A 2005, 109, 9747), we obtain a complete effective vibrational Hamiltonian for the entire amide system. Couplings between neighboring peptide units are obtained using the anharmonic vibrational Hamiltonian of glycine dipeptide (GLDP) at the BPW91/6-31G(d,p) level. Electrostatic couplings between non-neighboring units are calculated by the fourth rank transition multipole coupling (TMC) expansion, including $1 / R^{3}$ (dipoledipole), $1 / R^{4}$ (quadrupole-dipole), and $1 / R^{5}$ (quadrupole-quadrupole and octapole-dipole) interactions. Exciton delocalization length and its variation with frequency in the various amide bands are calculated. The simulated infrared amide I and II absorptions and CD spectra of 24 residue $\alpha$-helical motifs $\left(\mathrm{SPE}_{3}\right)$ are in good agreement with experiment.
\end{abstract}

\section{Introduction}

The amide I, II, III, and A vibrational modes of proteins have distinct infrared absorption band shapes. ${ }^{1,2}$ Their sensitivity to hydrogen bonding, dipole-dipole interactions, and peptide backbone geometry provides useful indicators of secondary structural changes. ${ }^{3-7}$ The amide I and A modes originate from the stretching motion of the $\mathrm{C}=\mathrm{O}$ and $\mathrm{N}-\mathrm{H}$ bond, respectively, whereas the amide II and III are attributed to bending motion of the $\mathrm{N}-\mathrm{H}$ coupled to $\mathrm{C}-\mathrm{N}$ stretching. The vibrational circular dichroism (VCD) of the amide I, II, and III has been extensively applied to protein structure determination, ${ }^{8-12}$ and normal-mode analysis of ab initio calculations has been performed. ${ }^{13,14}$ However, most simulation effort has focused on the amide I vibrations ${ }^{15-20}$ which are highly localized (mostly $\mathrm{C}=\mathrm{O}$ stretch) and the easiest to model.

Coherent ultrafast vibrational spectroscopy is a powerful new technique for probing molecular structure and dynamics in the condensed phase. ${ }^{21-26}$ The cross peak of the amide A and I stretch vibrations of a model system of an amide bond has been observed. ${ }^{27,28}$ That cross peak region is particularly interesting because the amide A vibration is not resolved in the linear infrared spectra due to the broad absorption of water; nonlinear infrared spectra thus provide the only window into this vibration. Simulating such cross peaks requires the modeling of the couplings among the various amide modes.

The key ingredients of the required force field are frequencies of the local amide states and their couplings between neighboring and non-neighboring amide units. Computing vibrational $\mathrm{CD}$ and nonlinear chiral tensor components also requires the transition magnetic dipole moments between local amide states. These can be obtained by atomic axial tensor and normal-mode calculations of the chiral unit peptide for different Ramachandran angles.

We developed an electrostatic DFT map (EDM) ${ }^{29,30}$ of the fundamental, diagonal and off-diagonal anharmonicity frequency fluctuations of all amide states in NMA. The map provides a first principle effective vibrational Hamiltonian, which includes the fundamental, overtone, combination frequencies, and transi-
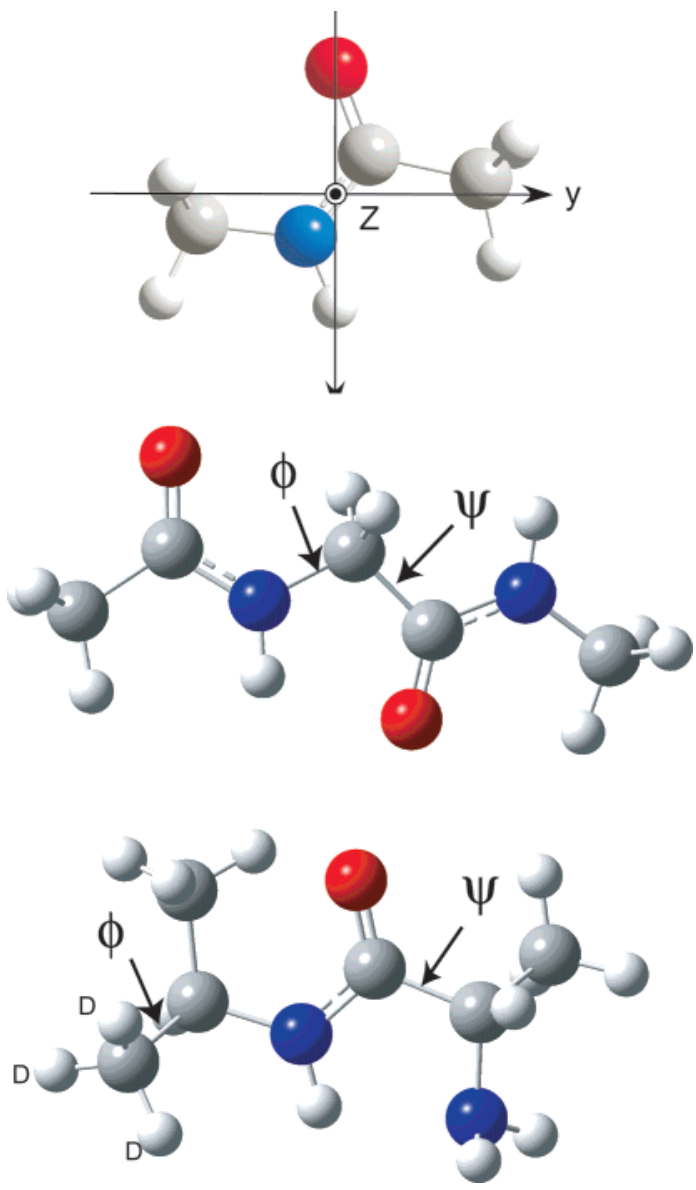

Figure 1. Cartesian coordinates of NMA used for constructing the local Hamiltonian and derivatives of the transition multipole moments (top), glycine dipeptide (GLDP) used for the neighboring coupling calculations (middle), and a unit peptide used for the magnetic dipole moment calculations (bottom).

tion moments. It further includes fourth-order derivatives of the electrostatic potential responsible for frequency and transition dipole fluctuations and line broadening. This Hamiltonian is in 
TABLE 1: Eigenfrequencies of NMA Amide Modes with Infinite Atomic Masses for the Two Methyl Groups Used for Constructing the Anharmonic Vibrational Hamiltonian of GLDP $^{a}$

\begin{tabular}{cccl}
\hline mode & frequency $/ \mathrm{cm}^{-1}$ & amide mode & \multicolumn{1}{c}{ description } \\
\hline 1 & $1207.9(1241.2)$ & amide III & $\mathrm{C}-\mathrm{N}$ str, $\mathrm{C}-\mathrm{N}-\mathrm{H}$ bend \\
2 & $1472.4(1506.6)$ & amide II & $\mathrm{C}-\mathrm{N}$ str, C-N-H bend \\
3 & $1722.2(1732.8)$ & amide I & $\mathrm{C}=\mathrm{O}$ str \\
4 & $3582.7(3582.8)$ & amide A & $\mathrm{N}-\mathrm{H} \mathrm{str}$
\end{tabular}

${ }^{a}$ Numbers in parentheses are the eigenfrequencies with the original atomic masses for two methyl groups.

good agreement with the recent experiment of the amide I and II cross peak. ${ }^{31}$

In this Article, we provide the other necessary ingredient of the effective Hamiltonian for larger peptides, the intermode couplings. Torii and Tasumi had constructed a map of amide I couplings between neighboring amide units starting with ab initio calculations of a glycine dipeptide (GLDP) for various Ramachandran angles $(\psi$ and $\phi)$ at the Hartree-Fock level. ${ }^{15}$ The couplings of the amide I fundamental between nonneighboring amide units were simulated using the transition dipole coupling mechanism (TDC). This TDC approach was recently extended to include the interaction of vibration-induced partial charges ${ }^{17}$ determined by normal-mode analysis of NMA. Contributions of the side chains to the amide I frequencies of $\beta$-hairpins were also investigated. ${ }^{18,19}$ Hydrogen-bonding interaction with the surrounding water was found to affect the amide I local-mode frequencies. These studies assumed bilinear couplings of the local amide modes (LAMs). Contributions of inter-amide couplings to the diagonal and off-diagonal anharmonicity of the amide modes were neglected.

We shall use a sixth-order expansion in local anharmonicities within the same unit. Coupling between neighboring units is taken to be quartic, and that between non-neighboring unit is assumed quadratic in the LAM. We then expand the potential in a basis of local amide states (LAS) consisting of the localized amide I, II, III, and A fundamental states and their overtone and combination states. The coupling matrix elements are calculated between all amide fundamentals, overtones, and combinations localized in different amide units. Couplings between neighboring amide units are modeled using a map constructed from the anharmonic vibrational Hamiltonian of glycine dipeptide (GLDP) at the DFT level. Non-neighboring couplings are simulated by the transition multipole coupling expansion (TMC) of the LAS. We have expanded the transition charge density couplings (TCDC) to fourth rank in multipoles which include $R^{-3}$ (dipole-dipole), $R^{-4}$ (dipole-quadrupole), and $R^{-5}$ (quadrupole-quadrupole and dipole-octapole) interactions. The higher order multipoles are required for a smaller $R$. Couplings of the overtones and combinations are calculated. Earlier studies considered only the couplings between the fundamental states..$^{15,17}$

The anharmonic amide vibrational Hamiltonian of a protein including the local Hamiltonian, vibrational coupling of neigh- boring amide units, and electrostatic vibrational couplings of non-neighboring units is given in section II. The Hamiltonian is recast in the local eigenstate basis in section III. The linear infrared and vibrational CD spectra are calculated in section IV. Our results are discussed in section V.

\section{The Anharmonic Vibrational Hamiltonian in Coordinate Space}

The vibrational Hamiltonian of a peptide expanded in local amide modes (LAM) is partitioned as

$$
\hat{H}=\hat{H}_{\text {local }}+\hat{H}_{\mathrm{I}}+\hat{H}_{\mathrm{II}}+\hat{H}_{\text {int }}
$$

where $\hat{H}_{\text {local }}$ is the local Hamiltonian for each amide bond, $\hat{H}_{\mathrm{I}}$ $\left(\hat{H}_{\mathrm{II}}\right)$ represent the couplings between the neighboring (nonneighboring) amide bonds, and $\hat{H}_{\text {int }}$ is the coupling with the radiation field. The local Hamiltonian is

$$
\begin{array}{r}
\hat{H}_{\text {local }}=\sum_{m}\left(\sum_{i} f_{m, i i}^{(2)}(\mathbf{C}) q_{m i}^{2}+\sum_{i, j, k} f_{m, i j k}^{(3)}(\mathbf{C}) q_{m i} q_{m j} q_{m k}+\ldots+\right. \\
\left.\sum_{i_{1}, \ldots, i_{6}} f_{m, i_{1} \ldots i_{6}}^{(6)}(\mathbf{C}) q_{m i_{1}} q_{m i_{2}} q_{m i_{3}} q_{m i_{4}} q_{m i_{5}} q_{m i_{6}}\right)
\end{array}
$$

where $m$ and $n$ label the amide bond site, and $q_{m i}, q_{m j}, \ldots$ are the five LAMs (amide III, II, I, and A modes and one mode related to $\mathrm{N}-\mathrm{H}$ bending and $\mathrm{C}-\mathrm{N}$ stretch at site $m^{30}$ ). This Hamiltonian is expanded to sixth order in local amide modes. The coefficients $f^{(n)}$ depend on an external electric field and its derivatives $\mathbf{C} \equiv\left(E_{x}, E_{y}, E_{z}, E_{x x}, E_{y y}, E_{z z}, \ldots\right)$ defined at the origin of the local frame fixed on the amide bond (Figure 1, top) generated by the solvent and protein. These were calculated by force calculations of NMA in different geometries at the DFT (BPW91/6-31G $(\mathrm{d}, \mathrm{p})^{32,33}$ ) level for different electrostatic components ${ }^{30}$ using a modified Gaussian 03 code. ${ }^{30,34}$ The fluctuating local Hamiltonian $\hat{H}_{\text {local }}(t)$ can be constructed by combining eq 2 and the trajectory of $\mathbf{C}(t)$ obtained from a MD simulation of peptides.

Neighboring couplings are expanded to quartic order in a LAM

$$
\begin{array}{r}
\hat{H}_{\mathrm{I}}=\sum_{m, n}^{|n-m|=1}\left(\sum_{i, j} J_{m n, i j}^{(2)}(\psi, \phi) q_{m i} q_{n j}+\sum_{i, j, k} J_{m n, i j k}^{(3)}(\psi, \phi) q_{m i} q_{n j} q_{n k}+\right. \\
\left.\sum_{i, j, k, l} J_{m n, i j k l}^{(4)}(\psi, \phi) q_{m i} q_{n j} q_{n k} q_{n l}\right)
\end{array}
$$

The coefficients $J^{(2)}, J^{(3)}$, and $J^{(4)}$ depend on the Ramachandran angles $\psi$ and $\phi$ between sites $n$ and $m$. They were obtained for optimized geometries of glycine dipeptide (GLDP) (Figure 1) on a $30^{\circ}$ grid of $\psi$ and $\phi$ varied between -180 and $+180^{\circ}$. The geometry optimzations did not converge at $(\phi, \psi)=(0,0)$, $(-30,0)$, or $(30,0)$ since these conformations are highly unstable due to the steric hindrance; these geometries, which are hardly

\begin{tabular}{|c|c|c|c|c|c|c|c|c|c|c|c|c|c|c|c|}
\hline mode & $\begin{array}{l}Q_{10} \\
\left(\mu_{z}\right) \\
\end{array}$ & $\begin{array}{l}Q_{11 \mathrm{c}} \\
\left(\mu_{x}\right) \\
\end{array}$ & $\begin{array}{l}Q_{11 \mathrm{~s}} \\
\left(\mu_{y}\right)\end{array}$ & $Q_{20}$ & $Q_{21 \mathrm{c}}$ & $Q_{21 \mathrm{~s}}$ & $Q_{22 \mathrm{c}}$ & $Q_{22 \mathrm{~s}}$ & $Q_{30}$ & $Q_{31 \mathrm{c}}$ & $Q_{31 \mathrm{~s}}$ & $Q_{32 \mathrm{c}}$ & $Q_{32 \mathrm{~s}}$ & $Q_{33 \mathrm{c}}$ & $Q_{33 \mathrm{~s}}$ \\
\hline 1 & 0.000 & 0.074 & 0.018 & 0.798 & 0.000 & 0.000 & 1.636 & -0.354 & 0.000 & 2.638 & -3.132 & 0.000 & 0.000 & 8.496 & -9.686 \\
\hline 2 & 0.000 & -0.095 & 0.342 & -0.761 & 0.000 & 0.000 & 0.957 & -1.240 & 0.000 & -2.571 & 0.497 & 0.000 & 0.000 & 0.163 & -3.778 \\
\hline 3 & 0.000 & 0.260 & -0.416 & 0.658 & 0.000 & 0.000 & 0.470 & -2.077 & 0.000 & -0.225 & -5.148 & 0.000 & 0.000 & -3.541 & 2.511 \\
\hline 4 & 0.000 & 1.379 & 0.097 & -0.215 & 0.000 & 0.000 & -7.483 & -1.506 & 0.000 & 2.395 & -1.441 & 0.000 & 0.000 & 14.865 & 0.232 \\
\hline 5 & 0.000 & 0.126 & 0.003 & -0.643 & 0.000 & 0.000 & 0.877 & 0.296 & 0.000 & -4.113 & -0.091 & 0.000 & 0.000 & 3.847 & 3.162 \\
\hline
\end{tabular}
accessible at room temperarture, were ignored. All bond lengths and torsional and dihedral angles of all atoms which belong to

TABLE 2: First Derivatives of Regular Spherical Harmonics Components of the Charge Density of NMA with Respect to Our Five Normal Coordinates in Atomic Units ${ }^{a}$

${ }^{a}$ The first three columns correspond to dipole derivatives. 

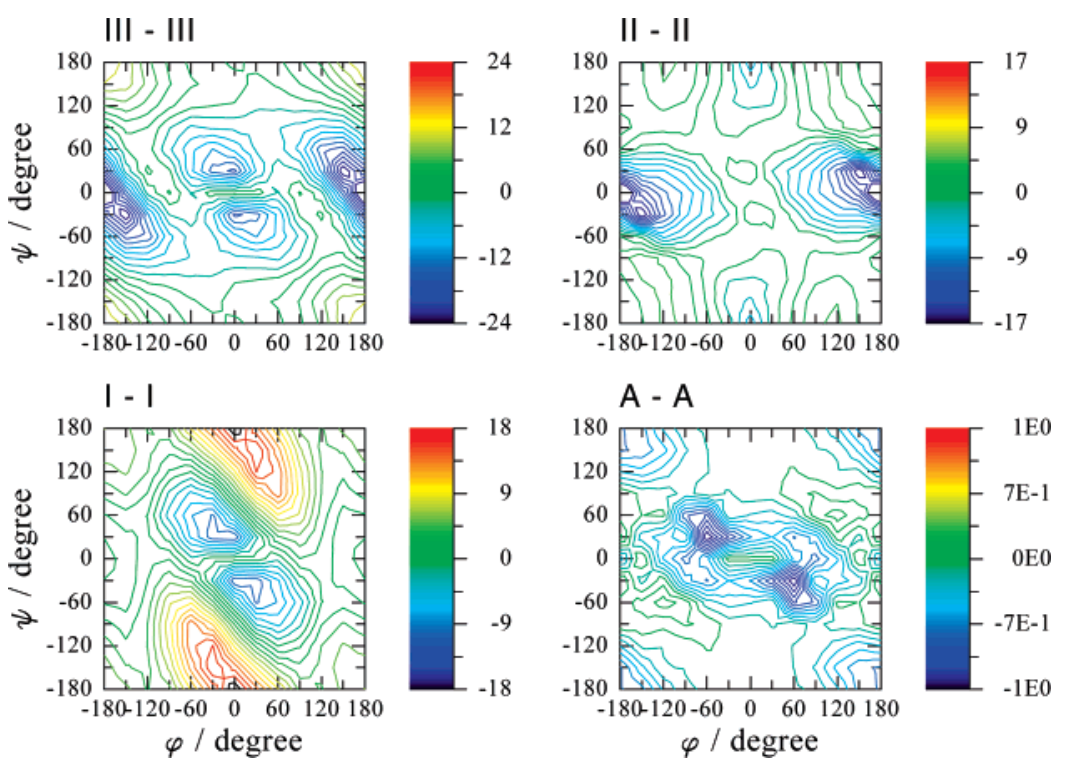

Figure 2. Coupling between two amide fundamentals in neighboring peptide units $\left(\hat{H}_{\mathrm{I}}\right)_{n a, n+1 a}$ versus Ramachandran angles (in $\left.\mathrm{cm}^{-1}\right)$.

TABLE 3: Regular Spherical Harmonics Components of the Transition Charge Density of NMA in Atomic Units ${ }^{a}$

\begin{tabular}{|c|c|c|c|c|c|c|c|c|c|c|}
\hline$a$ & $b$ & $Q_{11 \mathrm{c}}^{a b}\left(\mu_{x}\right)$ & $Q_{11 \mathrm{~s}}^{a b}\left(\mu_{y}\right)$ & $Q_{20}^{a b}$ & $Q_{22 \mathrm{c}}^{a b}$ & $Q_{22 \mathrm{~s}}^{a b}$ & $Q_{31 \mathrm{c}}^{a b}$ & $Q_{31 \mathrm{~s}}^{a b}$ & $Q_{33 \mathrm{c}}^{a b}$ & $Q_{33 \mathrm{~s}}^{a b}$ \\
\hline 0 & III & 0.0125 & -0.0485 & 0.1171 & -0.1565 & 0.2307 & 0.4362 & -0.0175 & 0.0905 & 0.5114 \\
\hline 0 & II & -0.0410 & 0.0711 & -0.1132 & -0.0626 & 0.3069 & 0.0078 & 0.8101 & 0.6033 & -0.4837 \\
\hline 0 & I & 0.0851 & 0.0052 & -0.0121 & -0.4560 & -0.0989 & 0.1439 & -0.1016 & 0.9034 & 0.0152 \\
\hline 0 & A & 0.0157 & 0.0004 & -0.0804 & 0.1095 & 0.0371 & -0.5137 & -0.0108 & 0.4799 & 0.3951 \\
\hline III & $\mathrm{III} \times 2$ & 0.0178 & -0.0693 & 0.1677 & -0.2222 & 0.3226 & 0.6231 & -0.0326 & 0.1135 & 0.7247 \\
\hline III & III + II & -0.0407 & 0.0704 & -0.1117 & -0.0652 & 0.3105 & 0.0131 & 0.8104 & 0.6039 & -0.4734 \\
\hline III & $\mathrm{III}+\mathrm{I}$ & 0.0851 & 0.0052 & -0.0121 & -0.4561 & -0.0988 & 0.1440 & -0.1014 & 0.9032 & 0.0157 \\
\hline III & $\mathrm{III}+\mathrm{A}$ & -0.0160 & 0.0006 & 0.0781 & -0.1065 & -0.0410 & 0.5058 & 0.0123 & -0.4794 & -0.4065 \\
\hline II & II $\times 2$ & -0.0581 & 0.1006 & -0.1587 & -0.0908 & 0.4335 & 0.0198 & 1.1455 & 0.8462 & -0.6899 \\
\hline II & $\mathrm{II}+\mathrm{III}$ & 0.0124 & -0.0486 & 0.1177 & -0.1553 & 0.2302 & 0.4380 & -0.0196 & 0.0906 & 0.5074 \\
\hline II & II + I & 0.0852 & 0.0051 & -0.0119 & -0.4561 & -0.0989 & 0.1441 & -0.1024 & 0.9027 & 0.0171 \\
\hline II & $\mathrm{II}+\mathrm{A}$ & 0.0148 & 0.0019 & -0.0828 & 0.1078 & 0.0447 & -0.5128 & 0.0079 & 0.4927 & 0.3857 \\
\hline I & $\mathrm{I} \times 2$ & 0.1203 & 0.0075 & -0.0173 & -0.6450 & -0.1393 & 0.2035 & -0.1423 & 1.2787 & 0.0212 \\
\hline I & I + III & 0.0124 & -0.0485 & 0.1171 & -0.1562 & 0.2308 & 0.4360 & -0.0173 & 0.0899 & 0.5115 \\
\hline I & I + II & -0.0411 & 0.0711 & -0.1132 & -0.0615 & 0.3071 & 0.0074 & 0.8103 & 0.6012 & -0.4837 \\
\hline I & $\mathrm{I}+\mathrm{A}$ & 0.0157 & 0.0004 & -0.0804 & 0.1098 & 0.0372 & -0.5139 & -0.0108 & 0.4793 & 0.3950 \\
\hline A & $\mathrm{A}+\mathrm{III}$ & -0.0125 & 0.0485 & -0.1173 & 0.1561 & -0.2305 & -0.4365 & 0.0184 & -0.0915 & -0.5099 \\
\hline A & $\mathrm{A}+\mathrm{II}$ & -0.0410 & 0.0711 & -0.1131 & -0.0625 & 0.3069 & 0.0079 & 0.8099 & 0.6032 & -0.4835 \\
\hline A & $\mathrm{A}+\mathrm{I}$ & 0.0851 & 0.0052 & -0.0121 & -0.4560 & -0.0989 & 0.1440 & -0.1014 & 0.9035 & 0.0151 \\
\hline
\end{tabular}

${ }^{a} Q_{10}^{a b}\left(\mu_{\mathrm{z}}\right), Q_{21 \mathrm{c}}^{a b}, Q_{21 \mathrm{~s}}^{a b}, Q_{30}^{a b}, Q_{32 \mathrm{c}}^{a b}, Q_{32 \mathrm{~s}}^{a b}$ are all zero and not included in the table.

two amide bonds ( 2 pairs of $\mathrm{H}, \mathrm{N}, \mathrm{C}, \mathrm{O}$ atoms) were constrained to have the same values as those in the optimized NMA geometry.

We first constructed two sets of localized amide modes of GLDP. The four local amide III, II, I, and A modes of one amide unit vibration are orthogonal to those of the other unit. The localized amide modes are obtained by diagonalizing the force constants of NMA with infinite masses for all atoms in the two methyl groups. All calculations were performed at the DFT (BPW91/6-31G(d,p)) level using the Gaussian 03 package. ${ }^{34}$ The localized amide mode frequencies are given in Table 1. The local amide A normal-mode frequency is virtually identical to that of NMA $\left(<0.1 \mathrm{~cm}^{-1}\right)$ since this mode is almost purely $\mathrm{N}-\mathrm{H}$ stretch and does not involve the methyl groups. The local amide $\mathrm{I}$ is also close to the NMA amide I frequency $\left(<10 \mathrm{~cm}^{-1}\right)$. The other bending vibrations (amide II and III) show larger deviations $\left(<30 \mathrm{~cm}^{-1}\right)$ due to the contribution of methyl vibrations. The potential of GLDP is expanded to fourth order in the eight LAM $q$ 's.

Through-space electrostatic interaction is assumed for couplings between non-neighboring peptide units. We start with the multipole expansion of the electrostatic energy

$$
\left(\hat{H}_{\mathrm{II}}\right)_{m n}=\sum_{l_{1}, l_{2}} \sum_{\kappa_{1}, \kappa_{2}} \delta \hat{Q}_{l_{m}{ } \kappa_{m}} \delta \hat{Q}_{l_{n}, \kappa_{n}} T_{l_{m} \kappa_{m}, l_{n} \kappa_{n}}
$$

where $\delta \hat{Q}_{l_{m}, \kappa_{m}} \equiv \hat{Q}_{l_{m}, \kappa_{m}}-\left\langle\hat{Q}_{l_{m}, \kappa_{m}}\right\rangle$, and $\hat{Q}_{l_{m}, \kappa_{m}}$ are the regular spherical harmonics operators of the charge density at site $m$, given by linear combinations of molecular multipole moments (see Table E.1 of ref 35). $T_{l_{1} \kappa_{1}, l_{2} \kappa_{2}}^{m n}$ are the interaction functions given in Appendix $\mathrm{F}$ of ref 35 , which depend on the relative positions of the two peptide units. The lowest three $(l=1,2$, and 3) regular spherical harmonics are defined with the local axis system on each amide unit and have 3, 5, and 7 terms. The three $l=1$ terms $\left(Q_{10}, Q_{11 \mathrm{c}}\right.$, and $\left.Q_{11 \mathrm{~s}}\right)$ represent the $\mu_{z}, \mu_{x}$, and $\mu_{y}$ components of the molecular dipole moment, respectively.

In the Born-Oppenheimer approximation, the multipoles depend parametrically on the LAMs. By taking the first order of the multipoles in local amide mode $q_{i}$, the coupling Hamiltonian becomes bilinear in the two local amide modes (see Appendix) 

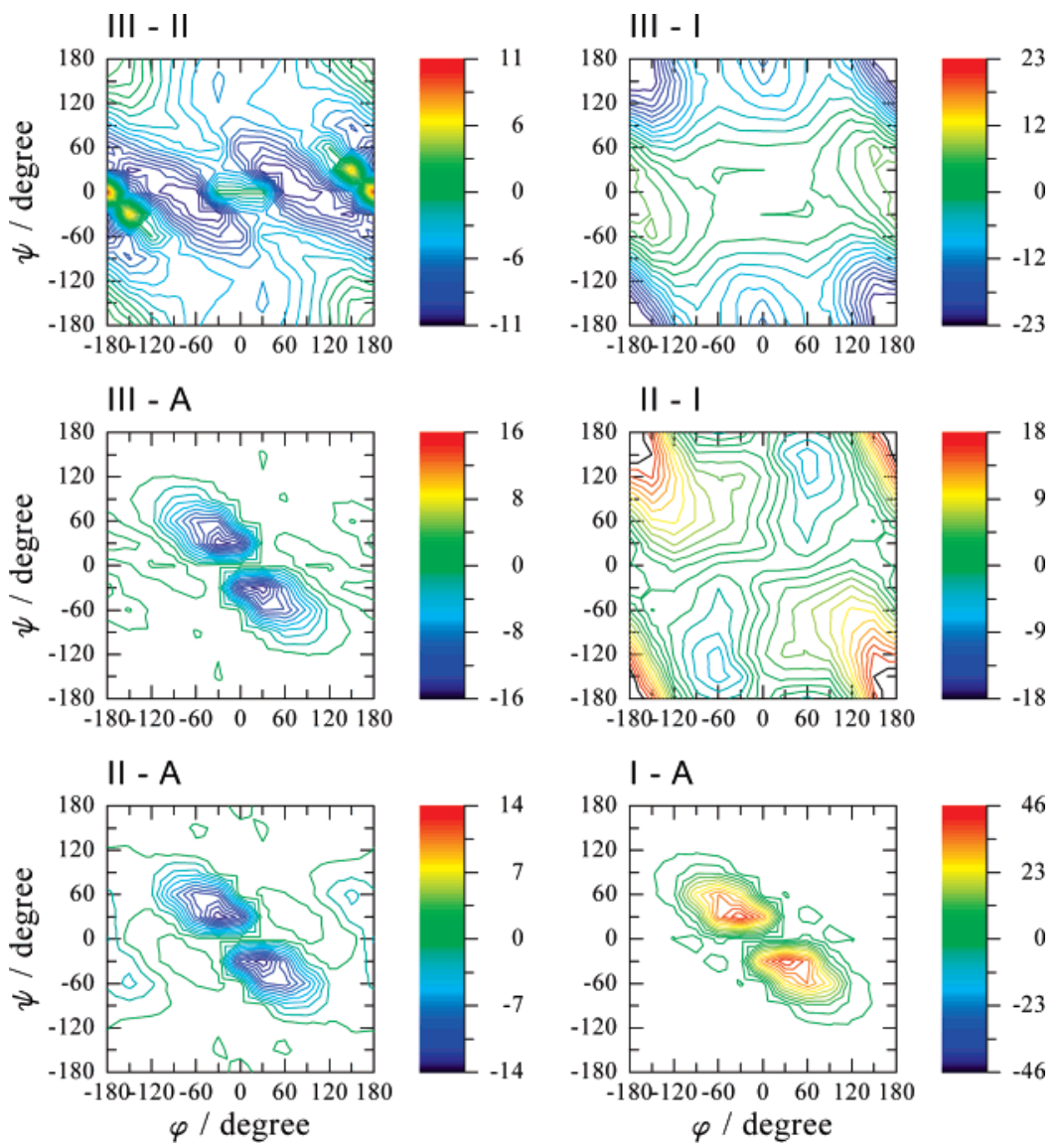

Figure 3. Coupling between two amide fundamentals in neighboring peptide units $\left(\hat{H}_{\mathrm{I}}\right)_{n a, n+1 b}$ versus Ramachandran angles (in $\left.\mathrm{cm}^{-1}\right)$.

$$
\hat{H}_{\mathrm{II}}=\sum_{m, n}^{|n-m| \geq 2} \sum_{i, j} K_{m n, i j} \hat{q}_{m i} \hat{q}_{n j}
$$

where

$$
K_{m n, i j}=\sum_{l_{1}, l_{2}} \sum_{\kappa_{1}, \kappa_{2}} \frac{\partial Q_{l_{1}, \kappa_{1}}}{\partial q_{m i}} \frac{\partial Q_{l_{2}, \kappa_{2}}}{\partial q_{n j}} T_{l_{1} \kappa_{1}, l_{2} \kappa_{2}}^{m n}
$$

The multipole expansion of the charge density couplings (eqs A4 and A5) gives an analytic expression for the potential, ${ }^{36}$ which depends on the molecular orientations with respect to a general coordinate frame. Accuracy can be improved by including higher multipoles, as needed.

Calculating the non-neighboring coupling (eqs 5 and 6) requires the first derivatives of the dipole, quadrupole, and octapole moments of NMA with respect to five normal coordinates (Table 1 of ref 30). These were calculated numerically at the same DFT level and in the same coordinate system (Figure 1) as the local vibrational Hamiltonian. They were converted to derivatives of regular spherical harmonics and reported in Table 2. The magnitude of the calculated dipole derivative $|\partial \mu / \partial Q|$ for the amide I normal mode is 1.38 in atomic units or $2.18 \mathrm{D} \AA^{-1} \mathrm{u}^{-1}$, compared to $2.73 \mathrm{D} \AA^{-1} \mathrm{u}^{-1}$ obtained by Torii and Tasumi. ${ }^{15}$

The interaction functions for the dipole-dipole couplings form a $3 \times 3$ matrix $^{35}$

$$
T_{\alpha \beta}^{m n}=R_{m n}^{-3}\left(3 r_{\alpha}^{m} r_{\beta}^{n}-c_{\alpha \beta}\right)
$$

where $\alpha, \beta=x, y, z$, and $R_{m n}$ is the distance between the two units. We took the origin at the middle point of the amide

hydrogen and oxygen (same as that in the origin of the electrostatic potential expansion in $\mathrm{EDM}^{30}$ ). The $r_{\alpha}^{m}$ is the product of the rotation matrix defining the local axis system of the $m$ 's unit and the unit vector in the direction from $m$ to $n$, and $c_{\alpha \beta}$ is the product of the two rotation matrices for $m$ and $n$.

The interaction functions for the higher order terms are similarly given in terms of the distance, unit vectors in the direction from $m$ to $n$, and the two rotation matrices defining the local axis systems (see Table F.1 of ref 35). Interaction functions for the dipole-quadrupole, quadrupole-quadrupole, and dipole-octapole interactions form $3 \times 5,5 \times 5$, and $3 \times$ 7 matrices and yield a $R_{m n}^{-4}, R_{m n}^{-5}$, and $R_{m n}^{-5}$, respectively.

The interaction functions only depend on the relative positions and orientations of the two peptide units; therefore, they need to be evaluated only once. We have truncated the expansion (eq 6) at rank $l_{1}+l_{2}=4$. These give $R_{m n}^{-3}$ (dipole-dipole), $R_{m n}^{-4}$ (quadrupole-dipole), and $R_{m n}^{-5}$ (quadrupole-quadrupole,) contributions to the potential. The higher multipoles are required when the two peptide units are close.

The coupling with the radiation field is

$$
\begin{aligned}
\hat{H}_{\mathrm{int}} & =\sum_{m} \sum_{i} q_{m i} \\
& \left(i \omega \frac{\partial \boldsymbol{\mu}}{\partial q_{m i}}-\omega\left(\boldsymbol{k} \cdot \boldsymbol{r}_{m}\right) \frac{\partial \boldsymbol{\mu}_{m}}{\partial q_{m i}}-\omega \frac{\partial \Phi_{m}}{\partial q_{m i}} \boldsymbol{k}-i \boldsymbol{k} \times \frac{\partial \boldsymbol{m}_{m}}{\partial q_{m i}}\right) \cdot \mathbf{A}(k, t)
\end{aligned}
$$

where $\mu_{m}$ and $\boldsymbol{m}_{m}$ are the electric and magnetic dipole moments, $\Phi_{m}$ is the electric quadrupole moment, $r_{m}$ is the position of the local amide frame origin at site $m$, and $\omega, \boldsymbol{k}$, and $\mathbf{A}$ are the frequency, wave vector, and vector potential of the radiation field. 

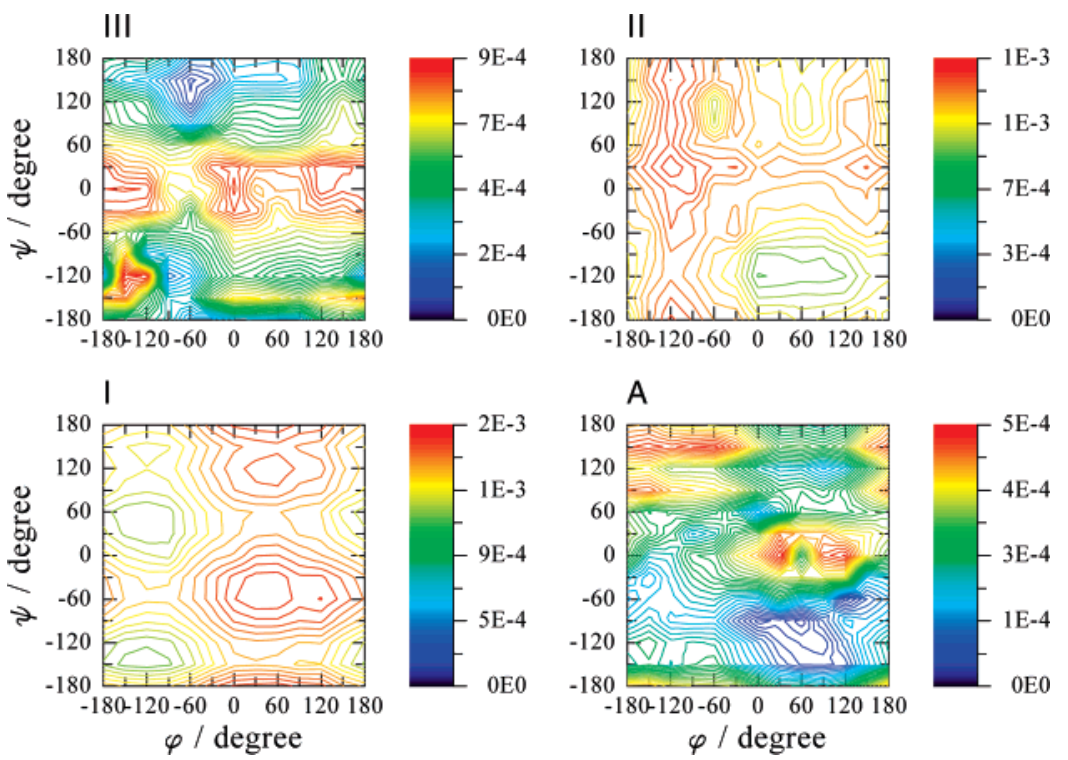

Figure 4. Variation of transition magnetic moments for amide III, II, I, and A modes ( $\boldsymbol{m}_{m a}$ in eq 17) with Ramachandran angles (in atomic units).
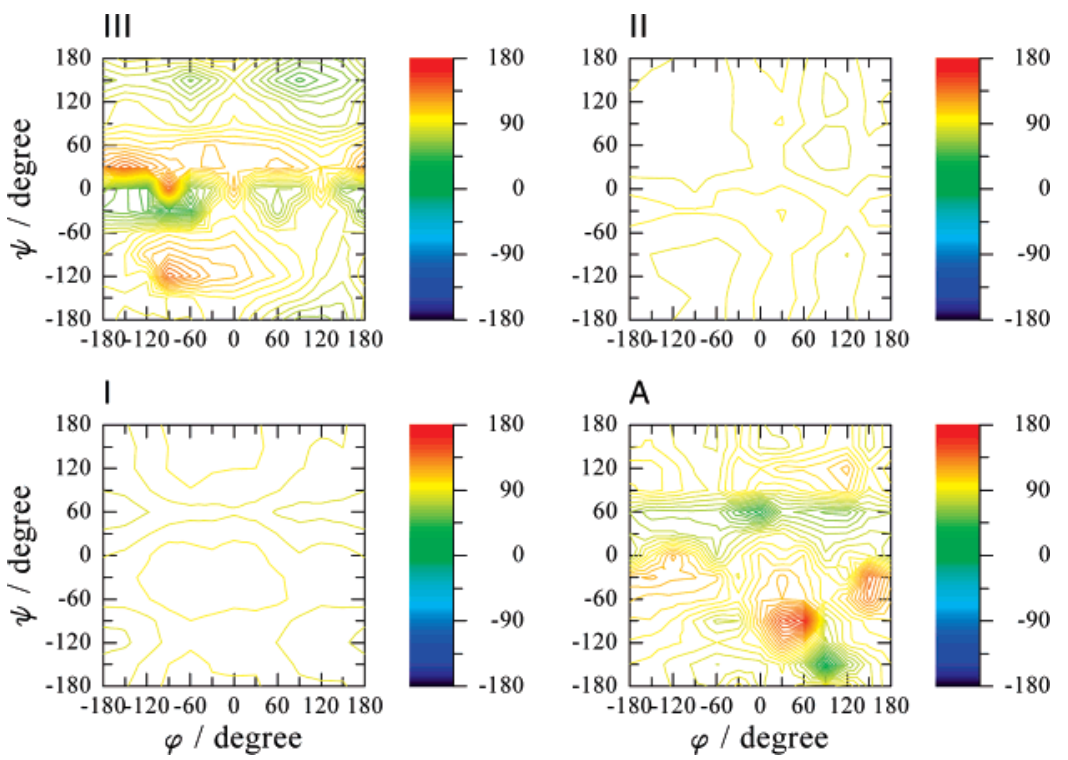

Figure 5. Angle between the magnetic and electric moments of amide III, II, I, and A modes ( $\boldsymbol{m}_{m a}$ and $\boldsymbol{\mu}_{m a}$ in eq 17) depicted versus the Ramachandran angles (in degrees).

Derivatives of magnetic moments with respect to the LAM depend on the two Ramachandran angles $\psi$ and $\phi$ of the unit peptide (Figure 1, bottom). The unit peptide has a similar structure to NMA (one amide bond) but is chiral since two hydrogens connected to the $\alpha$ carbon are substituted by methyl and $\mathrm{NH}_{2}$ groups and two hydrogens in the other methyl group are replaced by two methyl groups (one is deuterated). Geometry optimization and normal-mode calculations were carried out on a $30^{\circ}$ grid for $\psi$ and $\phi$. Magnetic moment derivatives were calculated based on the atomic axial tensor and the normal mode of each vibration. ${ }^{37}$

\section{Peptide Exciton Hamiltonian in the Local eigenstate Basis}

The local effective vibrational Hamiltonian (eq 2) of NMA was diagonalized to yield 14 eigenstates (ground state, 4 amide fundamentals (III, II, I, and A), first overtones of amide III, II, and I, and the combination states of amide III, II, and I) in the presence of a different electric field and its derivatives $\mathbf{C}$. The eigenfrequencies and transition dipole moments are reported as functions of $\mathbf{C}$ in ref 30. We employed these local amide states (LAS) as a basis set for the peptide Hamiltonian.

The local vibrational eigenstates of the $m$ th site (LAS) will be denoted as $|0\rangle,|m a\rangle$ ( $a$ runs over the 13 vibrational excited eigenstates excluding the ground state). We define the exciton creation and annihilation operators for LAS as $\hat{B}_{m a} \equiv|m a\rangle\langle 0|$ and $\hat{B}_{m a}^{\dagger} \equiv|0\rangle\langle m a|$. They satisfy the Pauli commutation relations

$$
\left[B_{m a}, B_{n b}^{\dagger}\right]=\delta_{n m} \delta_{a b}\left(1-\sum_{c} B_{m c}^{\dagger} B_{m c}\right)-\delta_{m n} \hat{B}_{m b}^{\dagger} \hat{B}_{m a}
$$

The local vibrational Hamiltonian is then written as

$$
\hat{H}_{\text {local }}=\sum_{m} \sum_{a} \hbar \omega_{m a}(\mathbf{C}) \hat{B}_{m a} \hat{B}_{m a}^{\dagger}
$$

The frequencies $\omega_{m a}$ of the 13 LAS, which depend on the electric field and its derivatives in the vicinity of the local amide bond (electrostatic DFT map), were reported earlier. ${ }^{30}$ The solvent peak shifts and bandwidths of linear infrared spectra of 

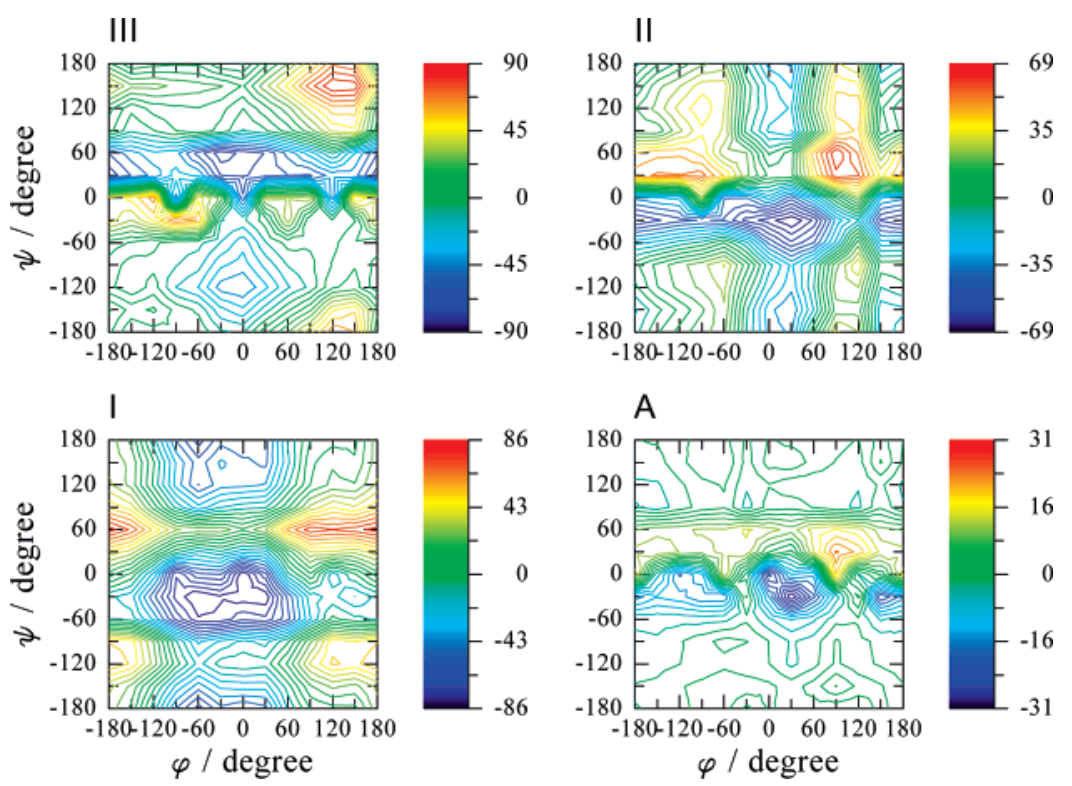

Figure 6. The rotational strength (eq 18) (in $\mathrm{esu}^{2} \mathrm{~cm}^{2} \times 10^{-44}$ ) of the amide III, II, I, and A modes shown versus the Ramachandran angles.

NMA calculated by combining the electrostatic DFT map and the trajectory of electrostatic components $\mathbf{C}$ generated by a MD simulation reproduced the experimental values.

To calculate neighboring couplings in the eigenstate basis, the Hamiltonian (eq 3) is expanded in a harmonic basis set $\mid N_{11}$, $\left.\ldots, N_{14} N_{21}, \ldots, N_{24}\right\rangle \equiv\left|N_{11}\right\rangle_{\ldots} .\left|N_{14}\right\rangle\left|N_{21}\right\rangle \ldots\left|N_{24}\right\rangle$, where $N_{n i}$ represent the $N$ th excited harmonic oscillator wavefunctions of the normal-mode $i$ in $n$th amide modes. Since one harmonic basis state dominates $(>95 \%)$ the eigenvector of each amide eigenstate (for example, the amide III fundamental (first overtone) eigenstates of unit 1 are dominated by the harmonic state $\left.\left|1_{11} 0 \ldots 0\right\rangle\left(\left|2_{11} 0 \ldots 0\right\rangle\right)\right)$, the couplings between the amide eigenstates were approximated as the couplings between the corresponding harmonic basis states. For example, the coupling between the amide III fundamental eigenstates of unit 1 and 2 is given by $\left\langle 1_{11} 0 \ldots 0\left|H_{\mathrm{I}}\right| 1_{11} 0 \ldots 0\right\rangle$.

Contour plots of the couplings between the same amide state in neighboring units $\left(H_{\mathrm{II}}\right)_{n a, n+1 a}$ with respect to $\phi$ and $\psi$ are shown in Figure 2. The amide I-amide I coupling has two regions, an antidiagonal negative region through $(\psi, \phi)=(0,0)$ and an antidiagonal positive region through $(\psi, \phi)=(-180,0)$. These agree with the earlier study, ${ }^{15}$ but the relative amplitude of the negative region is larger in our study. This can be ascribed to two differences in the calculations. First, our study was performed with higher level basis sets (BPW91/6-31+G(d,p) compared to RHF/6-31+G(d,p))), and second, our calculation includes anharmonicities instead of calculating bilinear couplings of amide I normal modes. The amide I couplings have a maximum value of $18 \mathrm{~cm}^{-1}$ around $(\psi, \phi)=(30,120)$, which can be rationalized by the parallel configuration of two amide I vibrations. Amide III-III and II-II couplings are in the ranges of -23 to 6 and -18 to $4 \mathrm{~cm}^{-1}$, respectively. Amide II couplings have minimum values around $(\psi, \phi)=(150,30)$, where the two $\mathrm{N}-\mathrm{H}$ bonds are parallel. The transition dipoles of two local amide III modes are perpendicular to the $\mathrm{N}-\mathrm{H}$ bonds and are in head-to-tail configuration, resulting in the largest negative value. The amide $\mathrm{A}-\mathrm{A}$ couplings have negative values along the antidiagonal line $\phi=\psi$ similar to those of amide I-I couplings, where two $\mathrm{N}-\mathrm{H}$ bonds are antiparallel. Amide A couplings are smaller than the coupling with the other three modes $\left(<1 \mathrm{~cm}^{-1}\right)$ since this vibration is localized on the $\mathrm{N}-\mathrm{H}$ bond $^{30}$ and has a smaller transition dipole moment (Table 3 ).

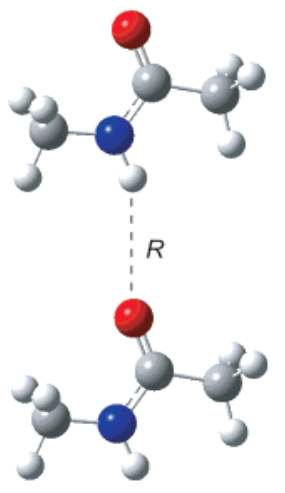

Figure 7. Structure of the NMA dimer used to calculate the variation of the electrostatic couplings between amide modes with the hydrogen bond length $R$ defined by the distance between oxygen and hydrogen.

Contour plots of couplings between different amide modes in neighboring units $\left(H_{\mathrm{II}}\right)_{n a, n+1 b}$ with respect to $\phi$ and $\psi$ are shown in Figure 3. The intermode couplings, $10-50 \mathrm{~cm}^{-1}$, are comparable to the intramode couplings. The amide III-I and amide II-I have the strongest couplings around $(\phi, \psi)=(180$,$180)$, where the two amide bonds are in-plane and the two transition dipole moments are parallel.

The through-space couplings between non-neighboring amide bonds $\hat{H}_{\text {II }}$ (eq 5) (see Appendix) are expanded as ${ }^{36,39-41}$

$$
\hat{H}_{\mathrm{II}}=\hat{H}_{1}+\hat{H}_{2}
$$

where

$$
\begin{gathered}
\hat{H}_{1}=\sum_{m, n}^{m \neq n}\left\{\sum_{a, b} K_{m n, a b}^{\prime}\left(\hat{B}_{m a}^{\dagger} \hat{B}_{n b}+\hat{B}_{m a} \hat{B}_{n b}^{\dagger}\right)+\right. \\
\left.\sum_{a, b, c, d} K_{m n, a b c d}^{\prime \prime \prime} \hat{B}_{m a}^{\dagger} \hat{B}_{m b} \hat{B}_{n c}^{\dagger} \hat{B}_{n d}\right\} \\
\hat{H}_{2}=\sum_{m, n}^{m \neq n}\left\{\sum_{a, b} K_{m n, a b}^{\prime}\left(\hat{B}_{m a} \hat{B}_{n b}+\hat{B}_{m a}^{\dagger} \hat{B}_{n b}^{\dagger}\right)+\right. \\
\left.\sum_{a, b, c} K_{m n, a b c}^{\prime \prime}\left(\hat{B}_{n a}^{\dagger} \hat{B}_{n b} \hat{B}_{m c}+\hat{B}_{n a}^{\dagger} \hat{B}_{n b} \hat{B}_{m c}^{\dagger}\right)\right\}
\end{gathered}
$$



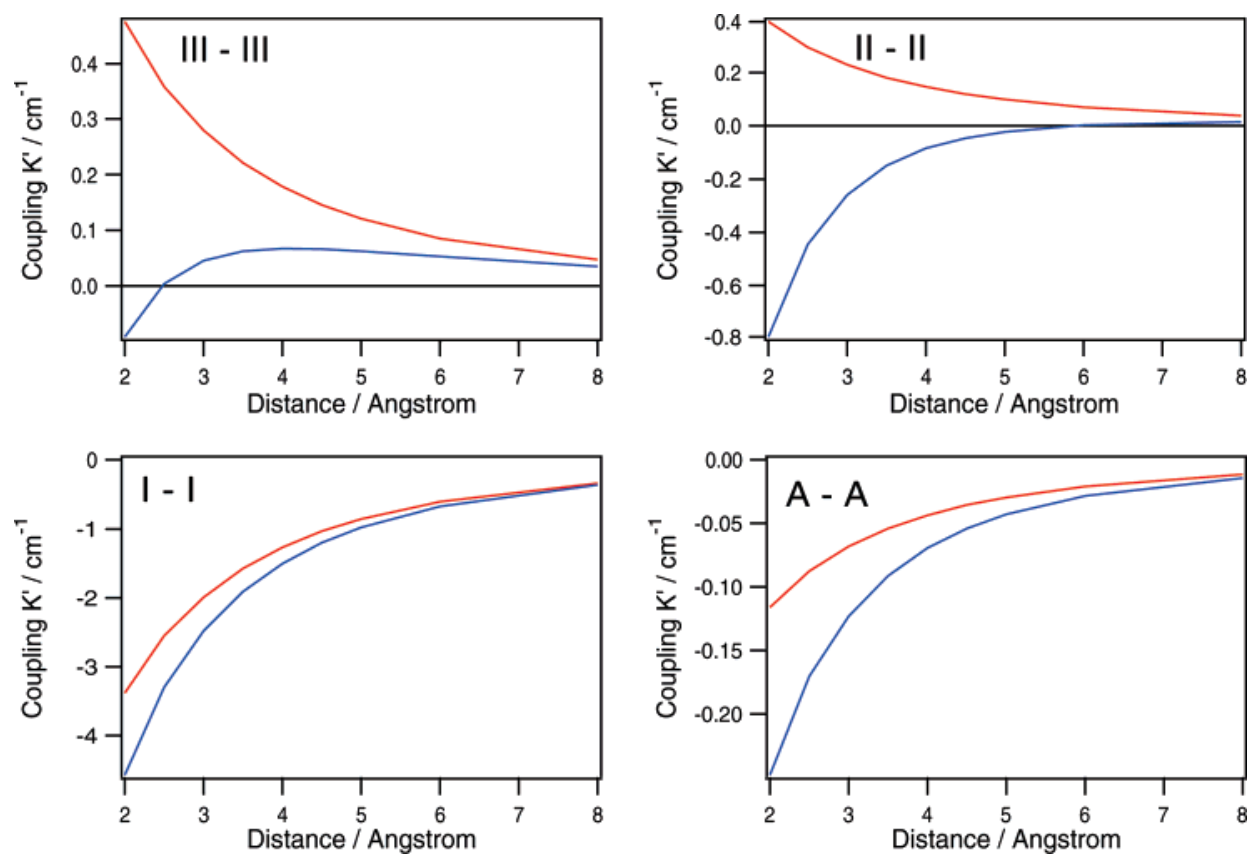

Figure 8. Dependence of the electrostatic couplings $K_{12, a a}^{\prime}$ (eq 14) between the amide III, II, I, and A modes of two NMA molecules on hydrogen bond length $(R$ in Figure 7$)$. Red: dipole-dipole interactions; blue: dipole-dipole, dipole-quadrupole, quadrupole-quadrupole, and dipoleoctapole interactions.

Both $\hat{H}_{1}$ and $\hat{H}_{2}$ include quadratic to quartic order terms in $B$ and $B^{\dagger}$. $\hat{H}_{1}$ conserves the number of excitations, but $\hat{H}_{2}$ does not. General expressions for $K^{\prime}, K^{\prime \prime}$, and $K^{\prime \prime \prime}$ are given in Appendix (eqs A8, A9, and A10). Expanding them in multipoles gives

$$
\begin{aligned}
& K_{m n, a b}^{\prime}=\sum_{l_{1}, l_{2}} \sum_{\kappa_{1}, \kappa_{2}} Q_{l_{1}, \kappa_{1}}^{0 a} Q_{l_{2}, \kappa_{2}}^{0 b} T_{l_{1} \kappa_{1}, l_{2} \kappa_{2}}^{m n} \\
& K_{m n, a b c}^{\prime \prime}=\sum_{l_{1}, l_{2}} \sum_{\kappa_{1}, \kappa_{2}} Q_{l_{1}, \kappa_{1}}^{a b} Q_{l_{2}, \kappa_{2}}^{0 c} T_{l_{1} \kappa_{1}, l_{2} \kappa_{2}}^{m n} \\
& K_{m n, a b c}^{\prime \prime \prime}=\sum_{l_{1}, l_{2}} \sum_{\kappa_{1}, \kappa_{2}} Q_{l_{1}, \kappa_{1}}^{a b} Q_{l_{2}, \kappa_{2}}^{c d} T_{l_{1} \kappa_{1}, l_{2} \kappa_{2}}^{m n}
\end{aligned}
$$

where $Q_{l_{1}, \kappa_{1}}^{a b}$ are the regular spherical harmonics components of the transition charge density calculated from eq A13.

Spherical harmonics components of the transition charge densities were calculated for all 105 transitions between our 14 states; 19 optically allowed transitions (4 from ground state to fundamental states, 3 from fundamentals to first overtones, and 12 from fundamental to combination states) have transition spherical harmonics larger than 0.001 au and are reported in Table 3. Other transitions were neglected.

In order to investigate the multipole contributions to the electrostatic interactions, the couplings between the amide III, II, I, and A modes of two NMA molecules (Figure 7) $K_{12, a a}^{\prime}$ were calculated with different hydrogen bond lengths. Calculations were done in two ways, using the dipole-dipole approximation and including dipole-dipole, quadrupole-dipole, quadrupole-quadrupole, and dipole-octapole interactions (Figure 8). The dipole-dipole contribution dominates the amide I coupling. The higher order multipole interactions are more significant for amide II, III, and A couplings.

The coupling with the radiation field in the eigenstate local basis is

$$
\begin{aligned}
\hat{H}_{\mathrm{int}}=\sum_{m} \sum_{a}\left(i \omega \boldsymbol{\mu}_{m a}-\omega\left(\boldsymbol{k} \cdot \boldsymbol{r}_{m}\right) \boldsymbol{\mu}_{m a}-\right. \\
\left.\omega \Psi_{m a} \boldsymbol{k}-i \boldsymbol{k} \times \boldsymbol{m}_{m a}\right) \cdot \mathbf{A}(k, t)
\end{aligned}
$$

where $\mu_{m a}$ and $\boldsymbol{m}_{m a}$ are the transition electric and magnetic dipole moments, $\Phi_{m a}$ is the transition electric quadrupole moment, and $r_{m}$ is the position of the amide units (the middle point of the amide hydrogen and oxygen).

Contour plots of the calculated transition magnetic moments for amide III, II, I, and A fundamentals with respect to $\psi$ and $\phi$ are shown in Figure 4. The transition magnetic moments for amide I have larger values when $\psi=-60$ and $120^{\circ}$ and $\phi=$ -120 and $60^{\circ}$. Amide III and A transition magnetic moments become larger as $\phi$ is close to $0^{\circ}$ due to stronger coupling between the amide $\mathrm{N}-\mathrm{H}$ and $\mathrm{NH}_{2}$ group. The amide II transition magnetic moment is large when $\psi$ is close to $-120^{\circ}$ and $\phi$ is close to $30^{\circ}$. Contour plots of angles between magnetic moment derivatives and electronic dipole moment derivatives for amide III, II, I, and A fundamentals with respect to $\psi$ and $\phi$ are shown in Figure 5. The transition magnetic and electric moments are close to perpendicular for the amide I and II fundamentals. The angles for amide III and A vary significantly (amide III: 47$141^{\circ}$, amide A: $57-140^{\circ}$ ) with $\psi$ and $\phi$. The rotational strength $R_{i}$ of the fundamental transition in the $i$ th normal mode is given by $^{37}$

$$
R_{i}=\hbar^{2} \operatorname{Im}\left[P_{i}^{\lambda}, M_{i}^{\lambda}\right]
$$

where

$$
P_{i \beta}^{\lambda}=\sum_{\alpha} P_{\alpha \beta}^{\lambda} S_{\lambda \alpha, i} \quad M_{i \beta}^{\lambda}=\sum_{\alpha} M_{\alpha \beta}^{\lambda} S_{\lambda \alpha, i}
$$

$P_{i \beta}^{\lambda}$ and $M_{i \beta}^{\lambda}$ are the atomic polar tensors and atomic axial tensors, and $S_{\lambda \alpha, i}$ is defined as

$$
X_{\lambda \alpha}=\sum_{i} S_{\lambda \alpha, i} Q_{i}
$$



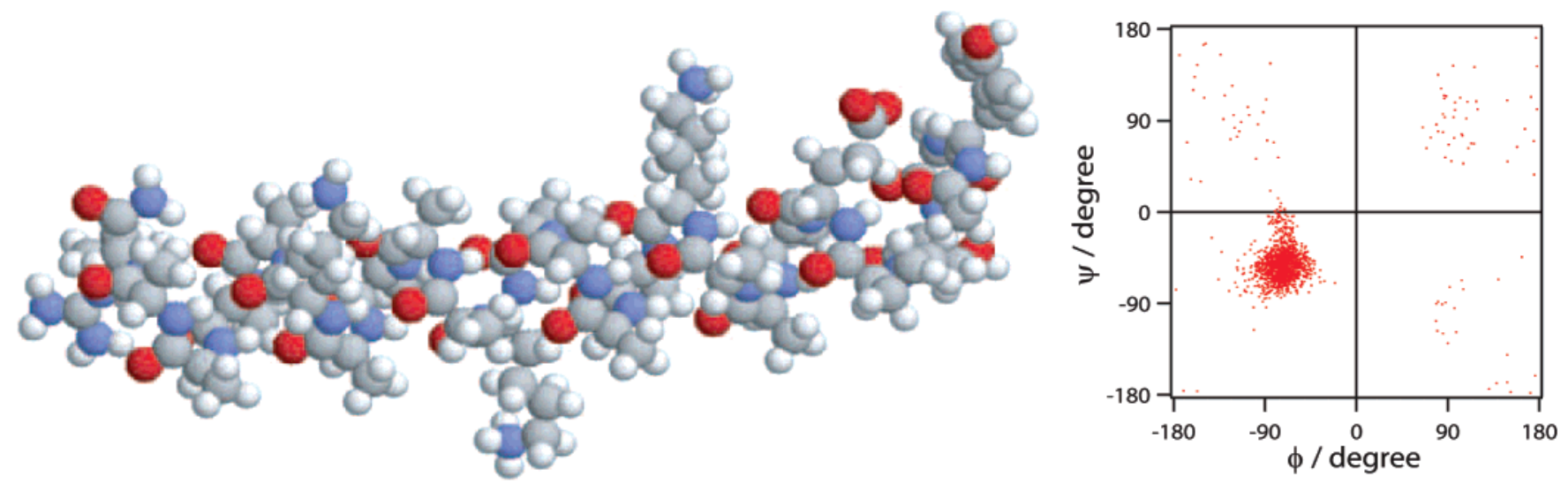

Figure 9. The $\alpha$-helical peptide $\mathrm{SPE}_{3}$. Top: 3D structure from a MD snapshot; bottom: distribution of Ramachandran angles obtained from 100 MD snapshots.
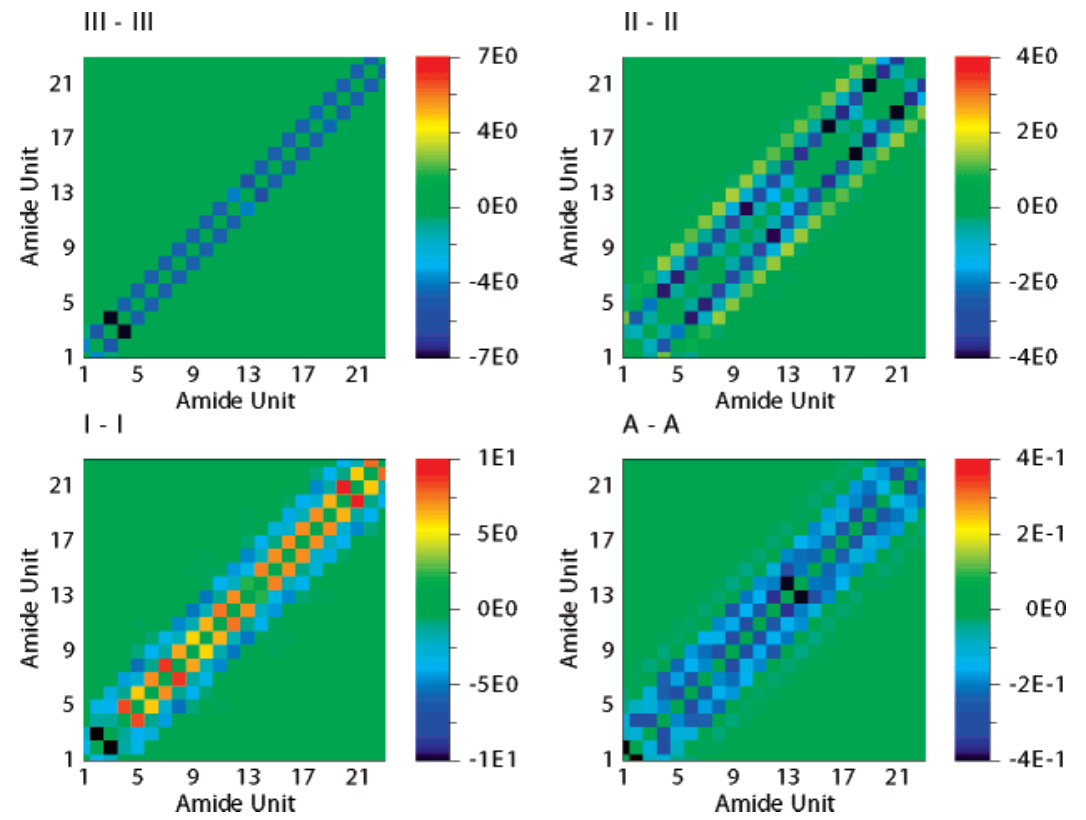

Figure 10. Vibrational couplings $K_{m n, a b}(\mathrm{eq} 5)\left(\right.$ in $\mathrm{cm}^{-1}$ ) between local fundamental amide states.

where $X_{\lambda \alpha, i}$ is the Cartesian displacement of the nucleus $\lambda$ and $Q_{i}$ is the normal coordinate. The rotational strengths of amide fundamentals are plotted in Figure 6. The dependence of amide I rotational strength on $\psi$ and $\phi$ is in good agreement with an earlier study. ${ }^{42}$ Due to the large variations of magnetic moment amplitudes and their angles with transition moments, the rotational strengths vary strongly and differently for each amide fundamental with $\psi$ and $\phi$. The rotational strengths are responsible for the vibrational $\mathrm{CD}$ spectra, and their strong dependence on Ramachandran angles suggests that vibrational CD signals should be very sensitive to the peptide secondary structures.

\section{Linear Absorption and Vibrational CD}

We have applied our effective Hamiltonian to $\mathrm{SPE}_{3}$, a 24 residue peptide (YGSPEAAA(KAAAA) ${ }_{3}$ r; $r$ represent D-Arg) known to have $\alpha$-helical structure. ${ }^{43}$ The fluctuating Hamiltonian is constructed for 100 snapshots obtained from a 2 ns MD trajectory generated earlier. ${ }^{44}$ Figure 9 shows the 3D structure of $\mathrm{SPE}_{3}$ from one MD snapshot and the distribution of Ramachandran angles. The Ramachandran angles are distributed around $(\phi, \psi)=(-70,-55)$ (in degrees), corresponding to a right-handed $\alpha$-helix, except for one amide bond connected to the terminal residue, which is distributed more uniformly. The 3D structure and the Ramachandran distribution confirms that $\mathrm{SPE}_{3}$ is conformationally stable in $\alpha$-helical structure, with a terminal residue rotating freely. Couplings of the amide I, II, III, and A fundamentals between different amide units are shown in Figure 10. Neighboring couplings are the largest. Nonneighboring amide III couplings are negligible. Amide I fundamentals have positive couplings over all regions, which can be attributed to the parallel configurations of transition dipoles. Due to the helical structure, the amide I dipoles of peptides $i$ and $i+3$ have a head-to-tail configuration and thus have negative couplings ( $\alpha$-helices have 3.6 residues per turn). The amide II and III fundamental couplings are negative due to their head-to-tail configuration. The amide II couplings between units $i$ and $i+4$ are positive. Amide A couplings are much weaker $\left(<1 \mathrm{~cm}^{-1}\right)$ than the other fundamentals since this mode has smaller transition dipoles and quadrupoles. Amide A vibrations are thus highly localized on each peptide unit.

The coefficients of each amide local mode to the 24 eigenstates of each amide III, II, I, and A fundamental in one MD snapshot are plotted in Figure 12. The amide II states are 

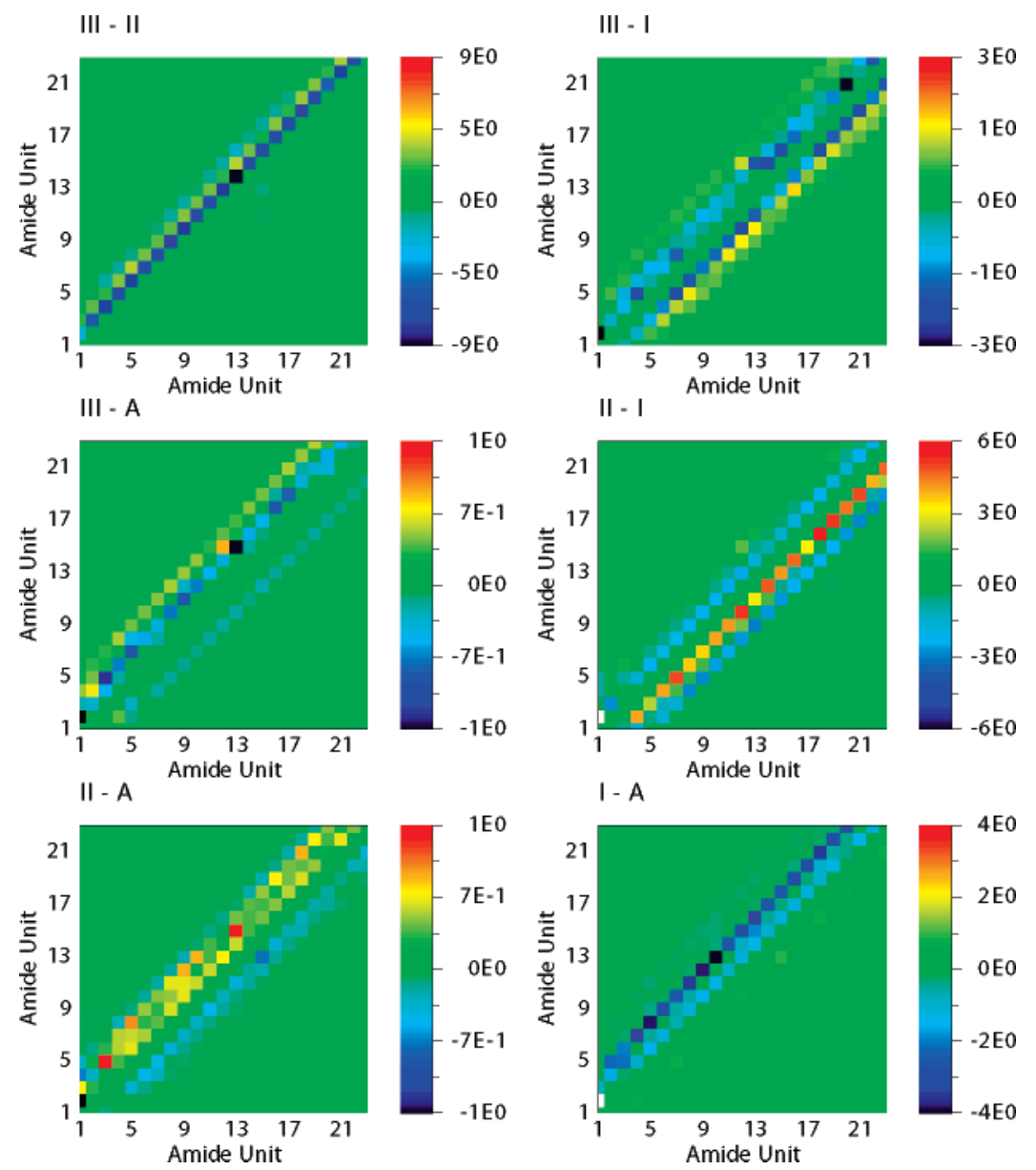

Figure 11. Vibrational couplings $K_{m n, a b}(\mathrm{eq} 5)\left(\right.$ in $\mathrm{cm}^{-1}$ ) between local fundamental amide states.
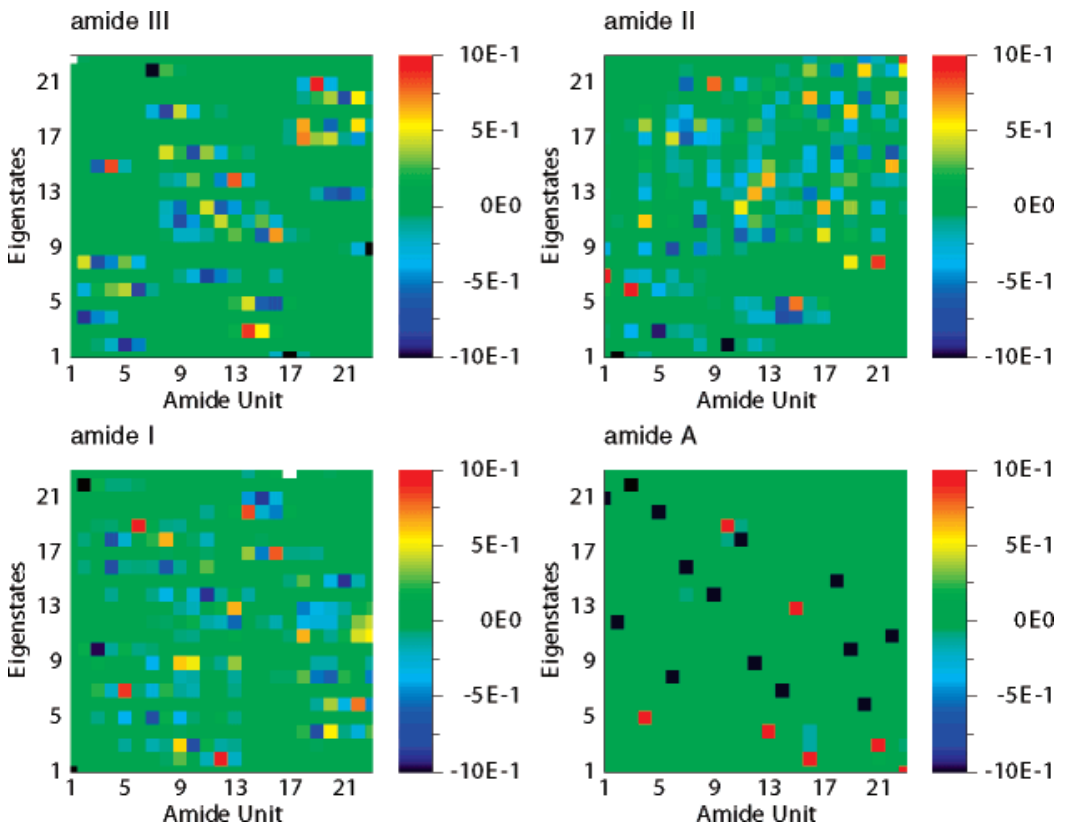

Figure 12. Contributions of each amide bond to the 24 amide III, II, I, and A fundamental eigenstates at one MD snapshot.

delocalized over almost the entire peptide chain. The amide III states are delocalized over neighboring amide bonds, but the amide I wave functions are delocalized along the helical axis (units $i$ and $i+3$ ).

The coherence length $L_{v}$ for eigenstates $v$ was calculated using the participation ratio $(\mathrm{PR})^{45-47}$

$$
L_{v}=\left(\sum_{m}\left(\sum_{a} C_{v, m a}^{2}\right)^{2}\right)^{-1}
$$

where $C_{v, m a}$ is the expansion coefficient of the $v$ eigenvector on LAS's $a$ at peptide unit $m$. The distributions of the PR binned over frequencies of the eigenstates in four amide fundamental regions are shown in Figure 14. In the amide I region, the lower 


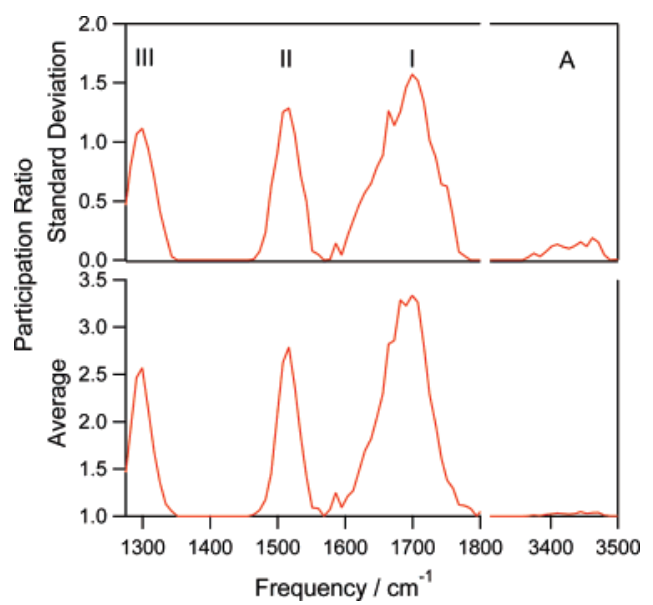

Figure 13. Distribution of the average and standard deviation of the participation ratio (PR) (eq 17) versus the frequency in the amide III, II, I, and A regions. The PR average is 1.606 (amide III), 2.350 (amide II), 1.761 (amide I), and 1.008 (amide A). The standard deviation is 0.494 (amide III), 1.227 (amide II), 0.533 (amide I), and 0.002 (amide A).

frequency eigenstates $\left(\sim 1600 \mathrm{~cm}^{-1}\right)$ are almost localized on one amide bond $(\mathrm{PR} \simeq 1)$; the eigenstates in higher frequencies are delocalized. In the amide III region, the higher frequency eigenstates $\left(\sim 1300 \mathrm{~cm}^{-1}\right)$ are localized. In the amide II region, there are two or three peaks in the PR distribution over all frequency ranges, which indicates the coexistence of multiple different delocalization states. Histograms of the average and standard deviation of the PR versus the frequency are calculated over 24 eigenstates assigned to each of 4 amide fundamentals and $100 \mathrm{MD}$ snapshots over 2 ns (Figure 13). The amide II fundamentals are most delocalized, with $L_{v}=2.3$, due to the larger neighboring couplings and transition moments and the smaller diagonal frequency fluctuations. The amide III and I fundamentals are also delocalized over 1.6 and 1.8 amide bonds. The amide A modes are highly localized due to the small transition moment and large diagonal frequency fluctuations.

Further exciton delocalization can be visualized by the following matrix ${ }^{45}$
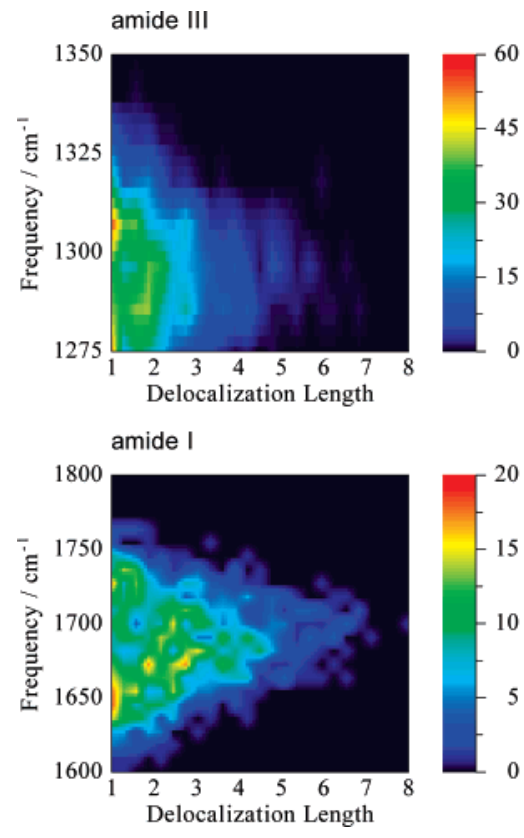

$$
\alpha_{n m}^{d}(\omega)=\left\langle\sum_{\nu} \sum_{a, b} \xi_{n a, m b}^{d} C_{v, n a} C_{v, m b}^{*} \delta\left(\omega-\omega_{v}\right)\right\rangle
$$

where $\xi_{\text {na,mb }}^{d} \equiv 1 / 3 \mu_{n a} \cdot \mu_{m b}$, and $\langle\ldots\rangle$ denotes an ensemble average. This is displayed for different frequency bins $\left(\int_{\omega_{0}}^{\omega_{1}} \mathrm{~d} \omega\right.$ $\left.\alpha_{n m}^{d}(\omega)\right)$ in the amide III, II, I, and A region in Figures 15, 16, 17, and 18. Delocalization along the diagonal (antidiagonal) axis represents the distribution of the exciton position (coherence) in real space. Excitons are more delocalized along the diagonal axis in the central frequency of each peak. They are more localized around the edges of the helix in the frequency bins of amide band tails. The amide III, II, and I excitons are delocalized significantly along the antidiagonal direction. Amide A excitons are localized on the diagonal axis. Exciton delocalization of amide III, II, and I bands significantly depend on the frequency bins. In $\alpha$-helical peptides, the backbone is always close to being perpendicular to the helical axis, making a spiral. For the amide I lower frequency region $\left(1664 \mathrm{~cm}^{-1}\right)$, the coherences are strong between units $i$ and $i+1$, reflecting the strong positive amide I couplings between the neighboring units. In the amide I higher frequency region $\left(1712 \mathrm{~cm}^{-1}\right)$, the coherence is strong between units $i$ and $i+3$, which are not directly covalently linked but have negative amide I couplings through hydrogen bonding along the helical axis. These suggests that the lower frequency amide I eigenstates are delocalized along the peptide chain, and the higher frequency states are delocalized along the helical axis.

Amide II excitons in the $1520 \mathrm{~cm}^{-1}$ region have coherence between the neighboring peptide units and also along the helical axis but have significant coherence between $i$ and $i+2$ around $1544 \mathrm{~cm}^{-1}$.

The infrared absorption and vibrational CD (VCD) spectra in the amide I and II region are shown in Figure 19 and compared with experiment on a typical $\alpha$-helix. ${ }^{48}$ In eq 17 , there are three interaction terms caused by the transition electric and magnetic moments and transition quadrupole moments. By symmetry, the quadrupole moment does not contribute to linear VCD spectra, which are determined by the transition electric and magnetic dipole moments. Calculations are performed with and without the transition magnetic moment $\left(-i \boldsymbol{k} \times \boldsymbol{m}_{m a}\right)$ in eq 17. Experimental CD band shapes in amide I are dispersive, and in II region, they are absorptive. The magnetic dipole is

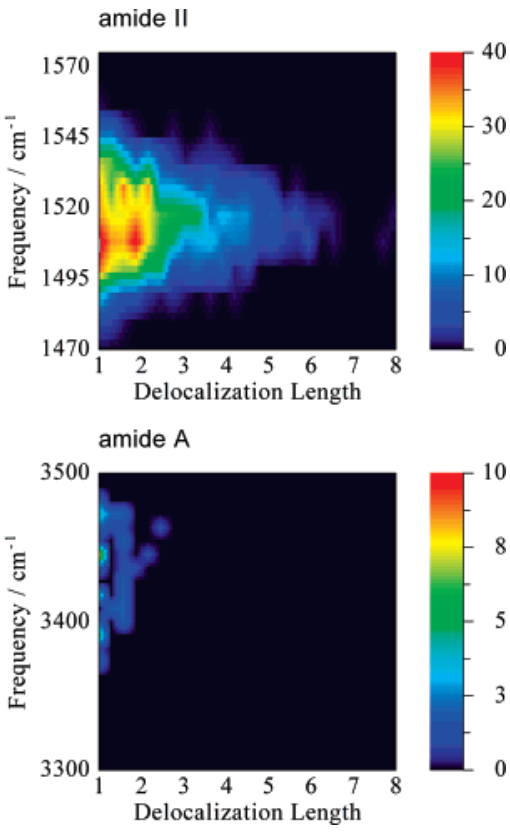

Figure 14. Distribution of the participation ration (PR) versus the frequency in the amide III, II, I, and A regions. 

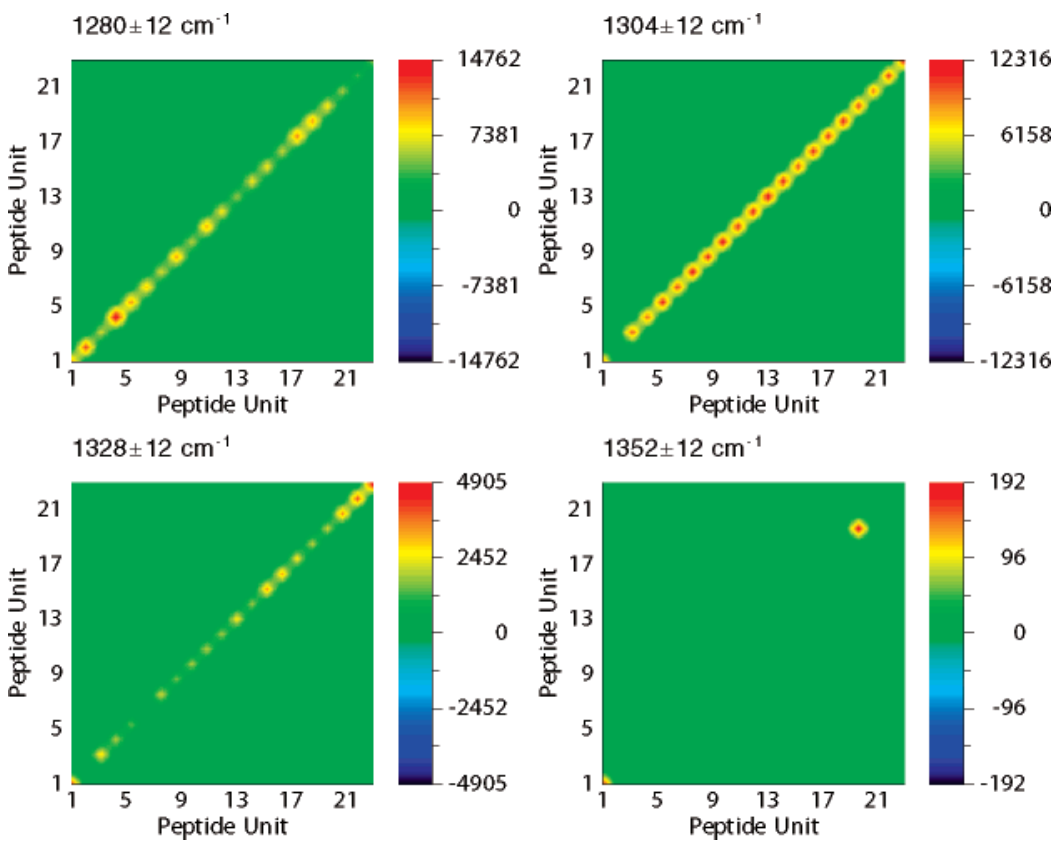

Figure 15. The exciton coherence (eq 21) for different frequency bins in the amide III region.
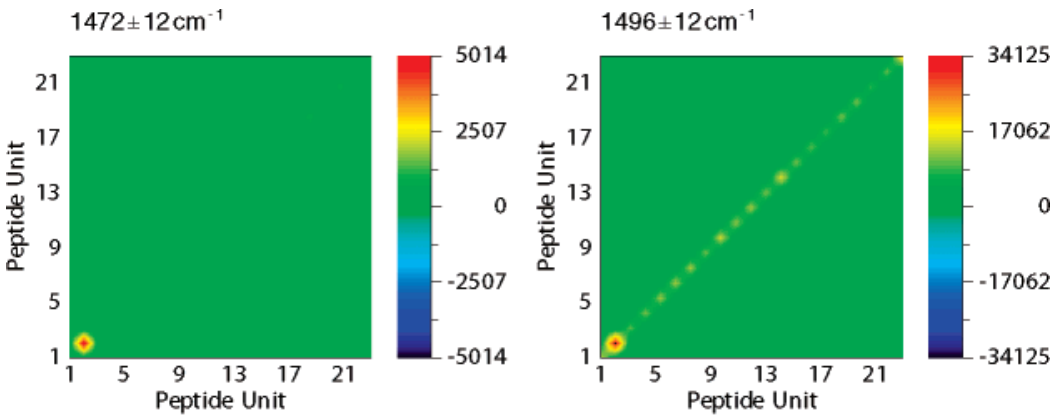
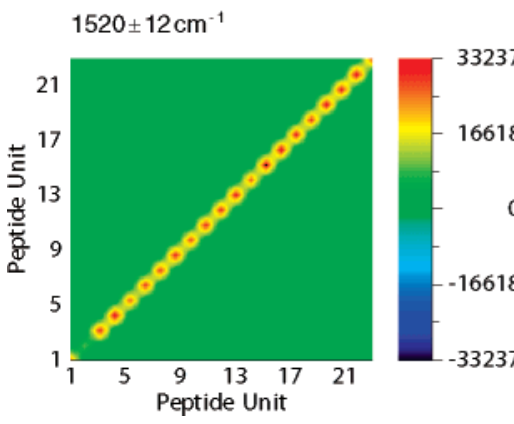

$1544 \pm 12 \mathrm{~cm}^{-1}$

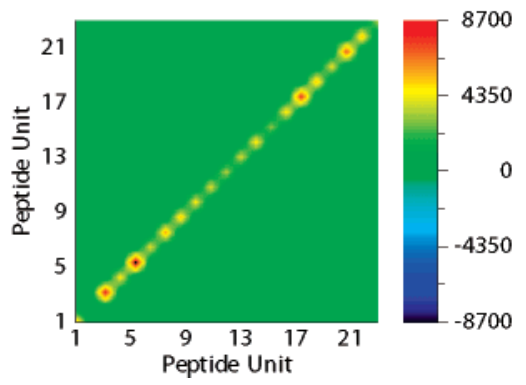

Figure 16. The exciton coherence (eq 21) for different frequency bins in the amide II region.

required to reproduce the absorptive band shape. Including the transition magnetic moment greatly improves agreement with experiment in the amide II region.

The infrared absorption and vibrational CD spectra in the amide III and A regions are displayed in Figure 20. The magnetic contribution of the peptide fragment to the amide III VCD peak is very weak, but it is significant in amide A, which does not have a simple dispersed line shape, but a more complicated profile.

The infrared absorption spectra are calculated with and without the couplings between different amide states in two neighboring peptide units (Figure 21). Intermode couplings between the neighboring units red shifted the amide I band by $10 \mathrm{~cm}^{-1}$.

\section{Discussion}

Using the LAS, Coulombic interactions can be expressed exactly using up to quartic (fourth) order expansion in the creation and annihilation operators. ${ }^{39}$ LAM expansions require higher order terms, which increases computational cost. Assuming through-space (Coulombic) interaction, transition charge density couplings (TCC) are expressed exactly up to fourth order in Pauli creation and annihilation operator of the LAS and can be exactly reproduced by the TMC expansion. TMC has a lower computational cost than the induced partial charge coupling model. ${ }^{17}$ In the latter, the accuracy is determined by the number of point charges $n$, and the number of required calculations of distances between charges scales as $n^{2}$. The TMC approach requires only a single calculation of distance for each pair of 

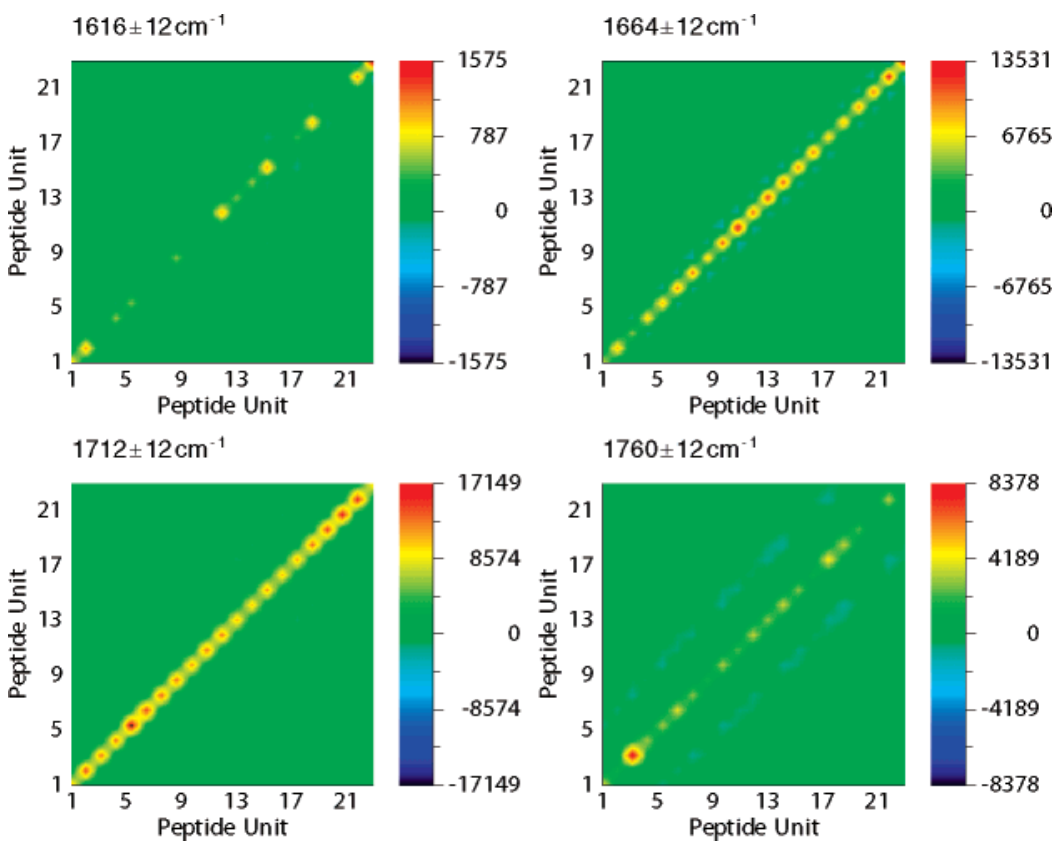

Figure 17. The exciton coherence (eq 21) for different frequency bins in the amide I region.
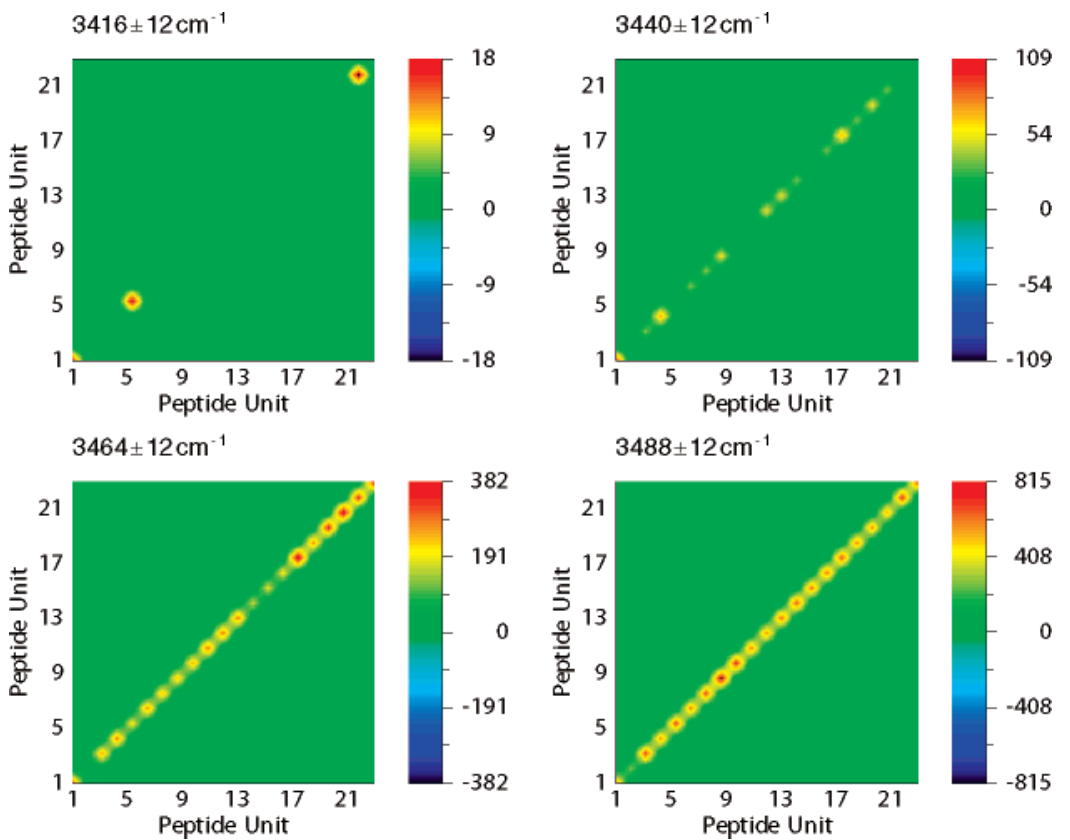

Figure 18. The exciton coherence (eq 21) for different frequency bins in the amide A region.

amide bonds. The TMC accuracy can be improved systematically by adding higher multipoles. There is no systematic way to determine the positions of point charges and improve the accuracy.

We have developed an effective vibrational Hamiltonian for the amide modes of peptides, including all amide modes. In previous studies, we constructed an electrostatic DFT map (EDM) for diagonal frequency fluctuations of local amide states (LAS), which includes four amide fundamentals, four overtones, and six combination states. Anharmonicity fluctuations are automatically built in. The LAS were calculated by diagonalizing the anharmonic vibrational Hamiltonian in the presence of an external nonuniform electric field. The calculation includes the change of the amide normal modes and the electron polarization in response to the external electric multipole field generated by solvent and other parts of the peptide. The EDM does not depend on a specific solvent and is transferable to any solvent. It is also straightforward to construct the map of deuterated NMA. The couplings between the neighboring amide units are calculated as functions of Ramachandran angles by ab initio calculation of glycine dipeptide (GLDP) at the same level as EDM. The transition multipole couplings (TMC) mechanism, including dipole-dipole, dipole-quadrupole, quadrupolequadrupole, and dipole-octapole couplings, between LAS is employed for non-neighboring amide units. The couplings between two NMA molecules were calculated by employing transition dipole couplings (TDC) and TMC; higher multipole contributions were found to be significant for amide modes II, III, and A.

The effective vibrational Hamiltonian was applied to the $\alpha$-helical peptide $\mathrm{SPE}_{3}$. We have estimated the coherence length of the amide exciton states by using the participation ratio (PR) 

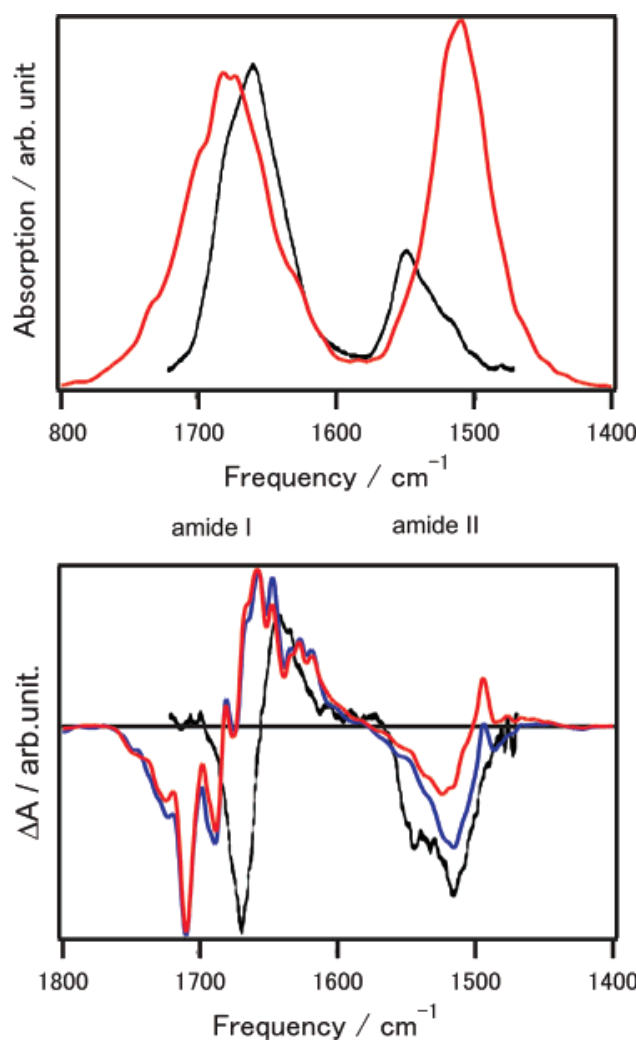

Figure 19. Vibrational CD spectra in the amide I and II regions. Blue: simulation with magnetic dipole moment; red: simulation without magnetic dipole moment; black: experiment. ${ }^{48}$
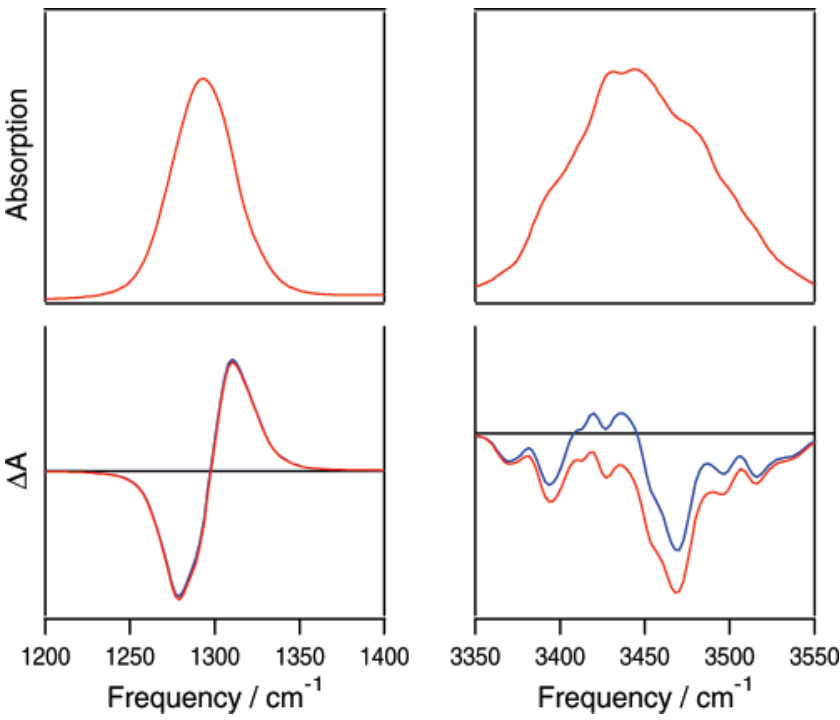

Figure 20. Vibrational CD spectra of amide III and A regions. Blue: simulation with magnetic dipole moment; red: simulation without magnetic dipole moment.

and found that the amide II states are most delocalized (PR = $2.35)$ and amide $\mathrm{A}$ is highly localized $(\mathrm{PR}=1.01)$. Contour plots of the exciton coherence in different frequency bins of each amide fundamental show the distribution of excitons and their coherence in real space. These strongly vary within each amide band. The amide I excitons have a large negative coherence between neighboring peptide units $(i$ and $i+1)$ at lower frequency and a larger negative coherence along the helical axis $(i$ and $i+3)$. This can be rationalized by the strong couplings of the parallel and head-to-tail configurations of transition dipoles, respectively. The negative coherence between the neighboring amide units in the low-frequency region of

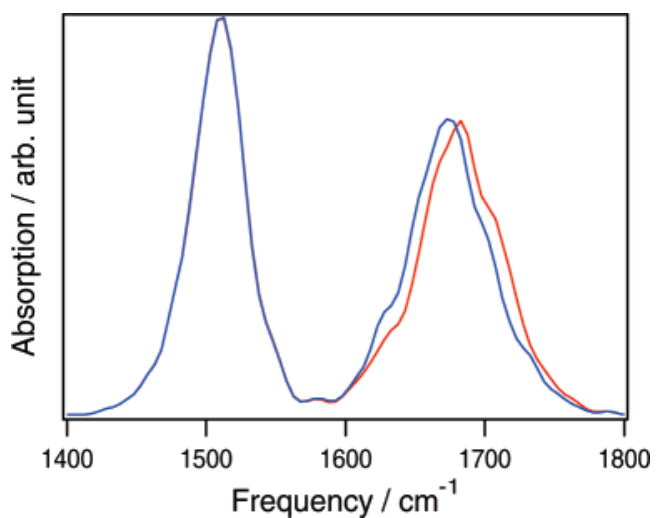

Figure 21. Calculated linear infrared absorption spectra with (blue) and without (red) intermode couplings between the neighboring units.

amide I is consistent with an earlier normal-mode study. ${ }^{14}$ That analysis of the $\alpha$-helical peptide labeled with $\mathrm{C}^{13}$ in two neighboring amide sites shows that the higher (lower) frequency mode is the out-of-phase (in-phase) linear combination of two localized amide I modes. This corresponds to the positive amide I coupling between the neighboring amide units and the stabilizing energy of two modes vibrating out-of-phase, which is consistent with our study. This is due to the fact that the two neighboring amide I vibrations are parallel.

The amide II excitons have stronger coherence between units $i$ and $i+2$, particularly in the higher frequency region. This can be rationalized since the amide II transition dipole moment lies in the amide plane and is almost parallel to the peptide backbone. The $i$ and $i+1$ dipoles are almost perpendicular (weak couplings), and $i$ and $i+2$ become antiparallel (strong negative couplings). The frequency dependence of the exciton coherence is different in various amide bands.

The linear infrared and vibrational CD spectra are calculated for all amide modes, and amide I and II band shapes are in good agreement with experiment. In our earlier study, we showed that the amide II and III vibrations are sensitive to a global electrostatic environment around the amide bond due to their delocalization. Consequently, their frequency fluctuations cannot be parametrized by the electrostatic potential at the four atom $(\mathrm{C}, \mathrm{O}, \mathrm{N}$, and $\mathrm{H})$ positions of the amide bond ${ }^{30}$ it can be difficult to apply earlier theoretical models ${ }^{17,19}$ to these bands. Amides II, III, and A provide promising sensitive probes for the peptide structures, which can be exploited by the recent progress in nonlinear vibrational spectroscopy.

Acknowledgment. We wish to thank Wei Zhuang for providing the MD trajectory of $\mathrm{SPE}_{3}$ and useful discussion. We also thank Dr. Darius Abramavicius for many helpful discussions regarding VCD spectroscopy. The support of the National Institutes of Health (Grant No. RO1 GM059230-05) and the National Science Foundation (Grant No. CHE-0446555) is gratefully acknowledged.

\section{Appendix: Through-Space Coupling}

In the Born-Oppenheimer approximation, the charge density $\rho_{m}$ on the amide bond $m$ is parametrized using the amide modes $q_{i}$ as

$$
\rho_{m}(\mathbf{r})=\rho_{m}^{(0)}(\mathbf{r})+\delta \rho_{m}\left(\mathbf{r} ; \mathbf{q}_{m}\right)
$$

where $\rho^{(0)}(\mathbf{r})$ represents the equilibrium charge density, and $\delta \rho$ $\left(\mathbf{r} ; \mathbf{q}_{m}\right)$ represents the charge density fluctuation induced by the $q_{i}$ vibration. When the charge density fluctuations at sites $m$ 
and $n$ do no overlap, the intercouplings are through-space (electrostatic)

$$
\left(H_{\mathrm{II}}\right)_{m n}=\sum_{i j} \int \mathrm{d} \mathbf{r} \int \mathrm{d} \mathbf{r}^{\prime} \frac{\delta \rho_{m}\left(\mathbf{r} ; \mathbf{q}_{i}\right) \delta \rho_{n}\left(\mathbf{r}^{\prime} ; \mathbf{q}_{j}\right)}{\mathbf{r}-\mathbf{r}^{\prime}}
$$

where the integration domains of $\mathbf{r}$ and $\mathbf{r}^{\prime}$ are localized in the vicinity of sites $m$ and $n$, respectively. We shall expand the charge density $\delta \rho_{m}\left(\mathbf{r} ; \mathbf{q}_{i}\right)$ to first order in $\mathbf{q}_{i}$

$$
\delta \rho_{m}\left(\mathbf{r}, \mathbf{q}_{i}\right)=\sum_{i} \frac{\partial \rho_{m}(\mathbf{r})}{\partial q_{i}} \hat{q}_{i}
$$

This gives

$$
\left(H_{\text {non-neighbor }}\right)_{m n}=\sum_{i j} K_{m n, i j} \hat{q}_{i} \hat{q}_{j}
$$

where

$$
K_{m n, i j}=\int \mathrm{d} \mathbf{r} \int \mathrm{d} \mathbf{r}^{\prime} \frac{\partial \rho_{m}(\mathbf{r})}{\partial q_{i}} \frac{\partial \rho_{m}\left(\mathbf{r}^{\prime}\right)}{\partial q_{j}}
$$

The charge density change at site $m$ can be expanded as

$$
\delta \rho_{m}(\mathbf{r})=\sum_{a, b} \delta \rho_{m}^{a b}|m a\rangle\langle m b|
$$

where $\delta \rho_{m}^{a b}=\langle m a|\delta \rho| m b\rangle$. Using these operators, we can write all operators in the vibrational space

$$
|m a\rangle\left\langle m b\left|=\hat{B}_{m a} \hat{B}_{m b}^{\dagger}(a, b \neq 0) \quad\right| 0\right\rangle\langle 0|=1-\sum_{a \neq 0} \hat{B}_{m a}^{\dagger} \hat{B}_{m a}
$$

Substituting these into eq A6, we note that the exact charge density change expanded in the operators $\hat{B}_{m a}$ and $\hat{B}_{m a}^{\dagger}$ only contains terms up to quadratic order

$$
\begin{array}{r}
\delta \rho_{m}=\delta \rho_{m}^{00}\left(1-\sum_{a \neq 0} \hat{B}_{m a}^{\dagger} \hat{B}_{m a}\right)+\sum_{a \neq 0}\left(\delta \rho_{m}^{0 a} \hat{B}_{m a}+\delta \rho_{m}^{a 0} \hat{B}_{m a}^{\dagger}\right)+ \\
\sum_{a \neq 0} \sum_{b \neq 0} \delta \rho_{m}^{a b} \hat{B}_{m a}^{\dagger} \hat{B}_{m b} \text { (A7) }
\end{array}
$$

By substituting $\delta \rho$ in eq A5 with eq A7 and since $\delta \rho_{m}^{00}=0$ by definition, we obtain eqs 11,12 , and 13 , where

$$
\begin{aligned}
& K_{m n, a b}^{\prime}=\int \mathrm{d} \mathbf{r} \int \mathrm{d} \mathbf{r}^{\prime} \frac{\delta \rho_{m}^{0 a}(\mathbf{r}) \delta \rho_{n}^{0 b}\left(\mathbf{r}^{\prime}\right)}{\left|\mathbf{r}-\mathbf{r}^{\prime}\right|} \\
& K_{m n, a b c}^{\prime \prime}=\int \mathrm{d} \mathbf{r} \int \mathrm{d} \mathbf{r}^{\prime} \frac{\delta \rho_{m}^{a b}(\mathbf{r}) \delta \rho_{n}^{0 c}\left(\mathbf{r}^{\prime}\right)}{\left|\mathbf{r}-\mathbf{r}^{\prime}\right|} \\
& K_{m n, a b c}^{\prime \prime \prime}=\int \mathrm{d} \mathbf{r} \int \mathrm{d} \mathbf{r}^{\prime} \frac{\delta \rho_{m}^{a b}(\mathbf{r}) \delta \rho_{n}^{c d}\left(\mathbf{r}^{\prime}\right)}{\left|\mathbf{r}-\mathbf{r}^{\prime}\right|}
\end{aligned}
$$

The LAM operator at site $m$ can also be expanded as

$$
\hat{q}_{m i}=\sum_{a, b} q_{m i}^{a b}|m a\rangle\langle m b|
$$

where $q_{m i}^{a b}=\left\langle m a\left|q_{m i}\right| m b\right\rangle$. The spherical harmonics operator of the charge density becomes

$$
\delta \hat{Q}_{l_{m}, \kappa_{m}}=\sum_{a, b} Q_{l_{m}, \kappa_{m}}^{a b}|m a\rangle\langle m b|
$$

where

$$
Q_{l_{m}, \kappa_{m}}^{a b} \equiv \sum_{i} \frac{\partial Q_{l_{m}, \kappa_{m}}}{\partial q_{m i}} q_{m i}^{a b}
$$

Similarly, we get

$\delta \hat{Q}_{l_{m}, \kappa_{m}}=\sum_{a \neq 0}\left(Q_{l_{m}, \kappa_{m}}^{0 a} \hat{B}_{m a}+Q_{l_{m}, \kappa_{m}}^{a 0} \hat{B}_{m a}^{\dagger}\right)+\sum_{a \neq 0} \sum_{b \neq 0} Q_{l_{m}, \kappa_{m}}^{a b} \hat{B}_{m a}^{\dagger} \hat{B}_{m b}$

Substituting in eq A14 into eq 4, we obtain eqs 14, 15, and 16.

\section{References and Notes}

(1) Miyazawa, T.; Shimanouchi, T.; Mizushima, S. I. J. Chem. Phys. 1958, 29,611

(2) Tsuboi, M.; Onishi, T.; Nakagawa, I.; Shimanouchi, T.; Mizushima, S. Spectrochim. Acta 1958, 12, 253.

(3) Torii, H.; Tasumi, M. J. Chem. Phys. 1992, 96, 3379.

(4) Torii, H.; Tasumi, M. Infrared Spectroscopy of Biomolecules; Wiley-Liss: New York, 1996.

(5) Byler, D. M.; Susi, H. Biopolymers 1986, 25, 469.

(6) Surewicz, W. K.; Mantsch, H. H. Biochim. Biophys. Acta 1988, 952, 115 . 329.

(7) Jackson, M.; Haris, P. I.; Chapman, D. J. Mol. Struct. 1989, 214,

(8) Keiderling, T. A. Nature 1986, 322, 851.

(9) Hilario, J.; Kubelka, J.; Keiderling, T. A. J. Am. Chem. Soc. 2003, 125,7562 .

(10) Yoder, G.; Keiderling, T. A.; Formaggio, F.; Crisma, M.; Toniolo, C. Biopolymers 1995, 35, 103 .

(11) Freedman, T. B.; Nafie, L. A.; Keiderling, T. A. Biopolymers 1995, $37,265$.

(12) Xie, P.; Zhou, Q. W.; Diem, M. J. Am. Chem. Soc. 1995, 117, 9502.

(13) Lee, S. H.; Krimm, S. Biopolymers 1998, 46, 283.

(14) Kubelka, J.; Huang, R.; Keiderling, T. A. J. Phys. Chem. B 2005, 109,8231

(15) Torii, H.; Tasumi, M. J. Raman Spectrosc. 1998, 29, 81.

(16) Moran, A. M.; Park, S.-M.; Mukamel, S. J. Chem. Phys. 2003, 118,9971

(17) Jansen, T. L.; Dijkstra, A. G.; Watson, T. M.; Hirst, J. D.; Knoester, J. J. Chem. Phys. 2006, 125.

(18) Hahn, S.; Ham, S.; Cho, M. J. Phys. Chem. B 2005, 109, 11789.

(19) Lee, H.; Kim, S. S.; Choi, J. H.; Cho, M. J. Phys. Chem. B 2005, $109,5331$.

(20) Bour, P.; Keiderling, T. A. J. Chem. Phys. 2003, 119, 11253.

(21) Asbury, J. B.; Steinel, T.; Stromberg, C.; Gaffney, K. J.; Piletic, I. R.; Goun, A.; Fayer, M. D. Chem. Phys. Lett. 2003, 374, 362.

(22) Bredenbeck, J.; Hamm, P. J. Chem. Phys. 2003, 119, 1569.

(23) Tanimura, Y.; Mukamel, S. J. Chem. Phys. 1993, 99, 9496.

(24) Kato, T.; Tanimura, Y. J. Chem. Phys. 2004, 120, 260.

(25) Saito, S.; Ohmine, I. J. Chem. Phys. 2003, 119, 9073.

(26) Nagata, Y.; Tanimura, Y. J. Chem. Phys. 2006, 124, 024508.

(27) Rubtsov, I. V.; Wang, J.; Hochstrasser, R. M. J. Chem. Phys. 2003, 118,7733 .

(28) Rubtsov, I. V.; Wang, J.; Hochstrasser, R. M. Proc. Natl. Acad. Sci. U.S.A. 2003, 100, 5601.

(29) Hayashi, T.; la Cour Jansen, T.; Zhuang, W.; Mukamel, S. J. Phys. Chem. A 2005, 109, 64

(30) Hayashi, T.; Zhuang, W.; Mukamel, S. J. Phys. Chem. A 2005 $109,9747$.

(31) DeFlores, L. P.; Ganim, Z.; Ackley, S. F.; Chung, H. S.; Tokmakoff, A. J. Phys. Chem. B 2006, 110, 18973.

(32) Perdew, J. P.; Chevary, J. A.; Vosko, S. H.; Jackson, K. A.; Pederson, M. R.; Singh, D. J.; Fiolhais, C. Phys. Rev. B 1992, 46, 6671.

(33) Becke, A. D. Phys. Rev. A 1988, 38, 3098.

(34) Frisch, M. J.; Trucks, G. W.; Schlegel, H. B.; Scuseria, G. E.; Robb, M. A.; Cheeseman, J. R.; Montgomery, J. A., Jr.; Vreven, T.; Kudin, K. N.; Burant, J. C.; Millam, J. M.; Iyengar, S. S.; Tomasi, J.; Barone, V.; Mennucci, B.; Cossi, M.; Scalmani, G.; Rega, N.; Petersson, G. A.; Nakatsuji, H.; Hada, M.; Ehara, M.; Toyota, K.; Fukuda, R.; Hasegawa, J.; Ishida, M.; Nakajima, T.; Honda, Y.; Kitao, O.; Nakai, H.; Klene, M.; Li, X.; Knox, J. E.; Hratchian, H. P.; Cross, J. B.; Bakken, V.; Adamo, C.; Jaramillo, J.; Gomperts, R.; Stratmann, R. E.; Yazyev, O.; Austin, A. J.; Cammi, R.; Pomelli, C.; Ochterski, J. W.; Ayala, P. Y.; Morokuma, K.; Voth, G. A.; Salvador, P.; Dannenberg, J. J.; Zakrzewski, V. G.; Dapprich, S.; Daniels, A. D.; Strain, M. C.; Farkas, O.; Malick, D. K.; Rabuck, A. D.; Raghavachari, K.; Foresman, J. B.; Ortiz, J. V.; Cui, Q.; Baboul, A. 
G.; Clifford, S.; Cioslowski, J.; Stefanov, B. B.; Liu, G.; Liashenko, A.; Piskorz, P.; Komaromi, I.; Martin, R. L.; Fox, D. J.; Keith, T.; Al-Laham, M. A.; Peng, C. Y.; Nanayakkara, A.; Challacombe, M.; Gill, P. M. W.; Johnson, B.; Chen, W.; Wong, M. W.; Gonzalez, C.; Pople, J. A. Gaussian 03; Gaussian, Inc.: Wallingford, CT, 2004.

(35) Stone, A. J. The Theory of Intermolecular Forces; Oxford University Press: Oxford, U.K., 1996; Vol. 32.

(36) Chernyak, V.; Zhang, W. M.; Mukamel, S. J. Chem. Phys. 1998, $109,9587$.

(37) Cheeseman, J. R.; Frisch, M. J.; Devlin, F. J.; Stephens, P. J. Chem. Phys. Lett. 1996, 252, 211.

(38) Hayashi, T.; Mukamel, S. J. Phys. Chem. A 2003, 107, 9113.

(39) Mukamel, S.; Berman, O. J. Chem. Phys. 2003, 119, 12194.

(40) Zhang, W. M.; Chernyak, V.; Mukamel, S. J. Chem. Phys. 1999, 110,5011 .
(41) Chernyak, V.; Mukamel, S. J. Opt. Soc. Am. B 1996, 13, 1302.

(42) Choi, J.-H.; Kim, J.-S.; Cho, M. H. J. Chem. Phys. 2005, 122, 174903.

(43) Wang, T.; Zhu, Y. J.; Getahun, Z.; Du, D. G.; Huang, C. Y.; DeGrado, W. F.; Gai, F. J. Phys. Chem. B 2004, 108, 15301.

(44) Zhuang, W.; Abramavicius, D.; Hayashi, T.; Mukamel, S. J. Phys. Chem. B 2006, 110, 3362.

(45) Piryatinski, A.; Asher, S. A.; Mukamel, S. J. Phys. Chem. A 2002, $106,3524$.

(46) Mukamel, S. Annu. Rev. Phys. Chem. 2000, 51, 691.

(47) Thouless, D. Phys. Rep. 1974, 13, 93.

(48) Kubelka, J.; Silva, R. A. G. D.; Keiderling, T. A. J. Am. Chem. Soc. 2002, 124, 5325. 